\title{
Co-loading of Levodopa and Curcumin Using Brain- targeted Protocells as a Drug Delivery System for Improving the Efficacy of Parkinson's Disease
}

wenkai zhou

Chinese Academy of Medical Sciences \& Peking Union Medical College Institute of Medicinal Biotechnology https://orcid.org/0000-0002-6589-6384

Chang Liu

Chinese Academy of Medical Sciences \& Peking Union Medical College Institute of Medicinal Biotechnology

Feifei Yu

Chinese Academy of Medical Sciences \& Peking Union Medical College Institute of Medicinal Biotechnology

\section{Xia Niu}

Chinese Academy of Medical Sciences \& Peking Union Medical College Institute of Medicinal

Biotechnology

\section{Xiaomei Wang}

Chinese Academy of Medical Sciences \& Peking Union Medical College Institute of Medicinal

Biotechnology

Guiling Li ( $\square$ liguiling@imb.pumc.edu.cn )

https://orcid.org/0000-0001-8585-6286

\section{Xinru Li}

Peking University School of Pharmaceutical Sciences

\section{Research}

Keywords: Parkinson's disease, Levodopa, Curcumin, Brain targeting, Lactoferrin, 58 Mesoporous silica nanoparticle, Protocells

Posted Date: June 29th, 2020

DOl: https://doi.org/10.21203/rs.3.rs-26742/v2

License: (9) This work is licensed under a Creative Commons Attribution 4.0 International License. Read Full License 

20 1 22

\section{Title page}

\section{Co-loading of Levodopa and Curcumin Using Brain-targeted} Protocells as a Drug Delivery System for Improving the Efficacy of

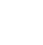

\section{Parkinson's Disease}

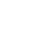

Wenkai Zhou ${ }^{1,2,4}$, Chang Liu ${ }^{1,2}$, Feifei $\mathrm{Yu}^{2}, \mathrm{Xia} \mathrm{Niu}^{2}$, Xiaomei Wang ${ }^{2}$, Guiling $\mathrm{Li}^{2 *}$, Xinru $\mathrm{Li}^{3 *}$

*Correspondence: liguiling@imb.pumc.edu.cn; 11@bjmu.edu.cn.

${ }^{1}$ Wenkai Zhou, Chang Liu contributed equally to this article.

${ }^{2}$ Institute of Medicinal Biotechnology, Chinese Academy of Medical Science \&

Peking Union Medical College, Beijing 100050, China.

${ }^{3}$ Department of Pharmaceutics, School of Pharmaceutical Sciences, Peking University, Beijing, China.

${ }^{4}$ Department of Immunology \& Centre for Immunotherapy, Institute of Basic Medical Sciences, Peking Union Medical College, Chinese Academy of Medical Sciences, Beijing, China.

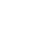
1 


\section{Co-loading of Levodopa and Curcumin Using Brain-targeted Protocells as a Drug Delivery System for Improving the Efficacy of Parkinson's Disease}

Abstract: Parkinson's disease (PD), one of the most common movement and neurodegenerative disorders, is challenging to treat, partly because the blood-brain barrier blocks passage of most drugs. Levodopa is a common clinical drug for controlling the symptoms of PD, but it only replenishes the missing dopamine, can't protect dopaminergic neurons. While curcumin as a neuroprotective agent has been reported for treatment of PD. Herein, we present a novel organic-inorganic composite nanoparticle with brain targeting (denoted as lf-protocells) for co-delivery of levodopa and curcumin, and demonstrate its attractive use as a biocompatible platform for PD treatment. The nanoparticle system is comprised of a lactoferrin (lf) modified lipid bilayer (LB) containing curcumin as its outer membrane and mesoporous silica nanoparticles (MSNs) containing levodopa as its supporting inner core. Our studies illustrate that the lf-protocells have a size of about $180 \mathrm{~nm}$ and spherical morphology, and can be used to co-load levodopa and curcumin efficiently. Further, a cell model and a mouse model induced by rotenone (Rot) and MPTP respectively are used to investigate the effects of binary-drug loaded lf-protocells on PD. Our results demonstrate that the combination of curcumin and levodopa alleviate the apoptosis of PD cells, enhance the cell viability as compared to levodopa used alone; levodopa together with curcumin also efficiently decrease the expression of a-synuclein, 
increase the expression of tyrosine hydroxylase in SH-SY5Y cells, and transform more levodopa into dopamine for supplement the loss of the brain. Moreover, the resulting binary-drug loaded lf-protocells ameliorate oxidative stress and mitochondrial dysfunction as compared to combination of free drugs. In addition, testing in a mouse model indicate that lf-protocells can improve significantly the motor function and distribution in brain compared with unmodified protocells. In conclusion, binary-drug loaded lf-protocells show much better therapeutic efficacy in both the cell model and the mouse model of PD and lower toxicity than bare MSNs. These results suggest that lf-protocells can be used as a promising drug delivery platform for targeted therapy against PD and other diseases of the central nervous system.

Keywords: Parkinson's disease, Levodopa, Curcumin, Brain targeting, Lactoferrin, Mesoporous silica nanoparticle, Protocells

\section{Background}

Parkinson's disease (PD) is the second most common chronic progressive neurodegenerative disorder after Alzheimer's disease (AD) as it affects almost 1-2\% of population aged over 65 in the world [1]. The typical pathological hallmarks of PD include the loss of dopaminergic neurons in the substantia nigra pars compacta (SNc) and the appearance of Lewy bodies formed by the accumulation of misfolded $\alpha$-synuclein ( $\alpha$-syn) protein. Although the pathogenesis of PD is still unknown [2], 
most reports show that it is associated with oxidative stress, neuro inflammatory reaction, mitochondrial dysfunction, cell apoptosis and so on [3-9].

So far, levodopa is considered the most effective medication for the symptomatic treatment of PD [10]. It works by being converted into dopamine and compensating for the dopamine deficiency in the brain. However, the efficacy of levodopa in the late stage of PD is significantly reduced due to its metabolism, subsequent low bioavailability and irregular fluctuations of its blood concentration [11]. Furthermore, levodopa, only as a neurotransmitter supplement, cannot effectively prevent or delay the deterioration of PD. It is noted that several drug candidates such as EPI-743, Baicalein and NTCELL currently used in clinical trials exert their therapeutic effects on PD by antioxidation, protecting mitochondria or acting on glial cells. It suggests that intervention of oxidative stress, improvement of mitochondrial dysfunction, resistance to neuroinflammation, protection of dopamine and other nerve cells are potential therapeutic strategies for PD [12].

As a natural polyphenol extracted from the dry rhizome of zingiberaceae plants [13], curcumin has numerous pharmacological properties such as anti-inflammation, antioxidation, anti-tumor and anti-nerve degeneration $[14,15]$. It reduces the high level of oxidative stress and neurotoxicity mainly through antioxidant effect and chelating metal ions, and thus achieves the protection of dopaminergic neurons and improves the level of dopamine [16-19]. Previous studies suggested that curcumin could be applied in the treatment of neurodegenerative diseases such as PD and AD $[20,21]$. Hence, if combined with levodopa, curcumin may lead to enhanced 
therapeutic effects of PD on the basis of levodopa supplementing neurotransmitters, since it works through antioxidation and repairing injured neurons. In other words, the combination of these two different durgs is expected to enhance the therapeutic effect of PD. So far, no relevant pharmacological study has been reported.

However, the physicochemical properties of hydrophilic levodopa and hydrophobic curcumin are quite different, which make their co-delivery confront with great challenges. It is necessary to construct an eligible drug delivery system that efficiently co-load these two drugs. Protocells, consisting of a mesoporous silica nanoparticles (MSNs) core coated by a layer of lipid bilayer (LB), are a class of highly scalable nanocarriers that have caused great concern in drug delivery applications $[22,23]$. Inner sponge-like inorganic MSNs with favorable surface area, high porosity and adsorption performance, extraordinary drug loading capacity, are excellent carriers of hydrophilic drugs such as levodopa. The outer membrane LB can not only contain hydrophobic drugs like curcumin but also effectively seal the surface pores of the inner MSNs to prevent drug leakage, thus achieving the co-delivery of the different drugs [23-27]. In addition, these drugs can be delivered simultaneously without affecting each other.

In our study, protocells delivery system co-loaded with levodopa and curcumin were constructed. In addition, brain targeting ligand-lactoferrin (Lf) was modified on the LB surface to enhance cellular uptake and brain targeting [28]. The blood-brain barrier $(\mathrm{BBB})$, formed by brain vessel endothelial cells linked together with tight junctions. This cellular interface helps to maintain a steady, optimal environment for 
neuronal function, and selective transport systems regulate the transport of wanted molecules. But on the other hand, it presents a major obstacle to the delivery of drugs passing from the bloodstream to the central nervous system (CNS) [29]. Lf receptor existing on the BBB mediated endocytosis is among the most efficient cellular uptake pathway. The delivery system with Lf attachment may be more efficient under certain pathological conditions of the high expression of Lf receptor, such as PD [30] and AD $[31]$.

The physicochemical properties, efficacy in vitro, mechanism of action and preliminary pharmacodynamics in vivo of the drug delivery system named as binary-drug loaded lf-protocells (L/C-Lf-Pro) were investigated. These results are expected to offer a beneficial strategy in mitigating the adverse reaction of levodopa and improving the therapeutic effect, and have the potential to provide a new approach for the development of drug delivery system with high-efficiency and important application prospect for curing PD.

\section{Materials and methods}

\subsection{Materials}

Tetraethyl orthosilicate (TEOS, 98\%), cetyltrimethylammonium bromide $(\mathrm{CTAB},>$ 99\%), Brij-58, 5,5'-dithiobis-2-nitrobenzoic acid (DTNB) and 2-iminothiolane hydrochloride (Traut's reagent) were purchased from Aladdin-chemistry (Shanghai, China). Lactoferrin was obtained from China Peptides Co. (Shanghai, China). 1,3,5-trimethylbenzene (TMB) and curcumin were purchased 
3-(4,5-Dimethylthiazol-2-yl)-2,5-diphenyltetrazolium bromide (MTT) and rhodamine

123 (Rh123) were purchased from Sigma Aldrich (USA). Cy5-SE was from MedChemExpress (USA). Triton X-100 was from Beijing Innochem Science \& WST-8, GSH and GSSG Assay Kit, primary antibody dilution buffer, transfer buffer and BeyoECL Moon were purchased from Beyotime Institute of Biotechnology (Shanghai, China). DMEM/F-12 medium, fetal bovine serum (FBS), penicillin-streptomycin and phosphate buffer saline (PBS) were purchased from

1,2-distearoyl-sn-glycero-3-phosphoethanolamine-N-[methoxy(polyethyleneglycol)-2

1,2-distearoyl-sn-glycero-3-phosphoethanolamine- $\mathrm{N}$-[maleimide(polyethylene glycol)-2000] (DSPE-PEG 2000-MAL), dipalmitoyl phosphatidylcholine (DPPC) and cholesterol (Chol) were purchased from A.V.T. Pharmaceutical Co., Ltd (Shanghai, China). Rotenone (Rot), 1-methyl-4-phenyl-1,2,3,6-tetrahydropyridine (MPTP), borate-EDTA buffer, Rhodamine $\mathrm{B}$ isothiocyanate (RBITC), (3-Aminopropyl) triethoxysilane (APTES) and SDS-PAGE running buffer were purchased from Dalian

151 Meilun Biotechnology Co., Ltd (Dalian, China). Levodopa (>98\%) was purchased from Beijing Innochem Science \& Technology Co., Ltd (Beijing, China). DAPI, 
were obtained from Beijing Solarbio Science \& Technology Co., Ltd (Beijing, China). Anti- $\beta$-actin, anti-GAPDH, anti- $\alpha$-synuclein and anti-tyrosine hydroxylase (TH) antibodies were obtained from Proteintech, USA. Anti-Bcl-2, anti-Bax, anti-Caspase-3 and anti-PARP antibodies were received from Cell Signaling Technology, USA. Secondary antibodies HRP-conjugated Affinipure Goat Anti-Mouse $\operatorname{IgG}(\mathrm{H}+\mathrm{L})$ and Anti-Rabbit $\operatorname{IgG}(\mathrm{H}+\mathrm{L})$ were obtained from Proteintech, USA. Deionized water was used in all experiments and analyses. All other chemicals were reagent grade and used directly without further purification or modification.

\subsection{Cell culture and treatments}

SH-SY5Y cells (purchased from the Ke Lei Biological Technology Co., Ltd.) were maintained in DMEM/F-12 medium containing 10\% fetal bovine serum (FBS), $1 \%$ antibiotic cocktail of penicillin-streptomycin at $37{ }^{\circ} \mathrm{C}$ under an atmosphere of $5 \%$ $\mathrm{CO}_{2}$ and $90 \%$ relative humidity.

The SH-SY5Y cells were subsequently divided into 8 experimental groups: (1) the control group: DMEM/F-12 medium alone, (2) the rotenone group: rotenone solution alone (Rot), (3) the curcumin group: $2 \mu \mathrm{M}$ curcumin (C) + Rot, (4) the levodopa group: $40 \mu \mathrm{M}$ levodopa (L) + Rot, (5) the levodopa and curcumin group: (40 $\mu \mathrm{M}$ levodopa $+2 \mu \mathrm{M}$ curcumin) (free L/C) + Rot, (6) the curcumin-loaded lf-protocells group: $2 \mu \mathrm{M}$ curcumin-loaded lf-protocells (C-Lf-Pro) + Rot, (7) the levodopa-loaded lf-protocells group: $40 \mu \mathrm{M}$ levodopa-loaded lf-protocells (L-Lf-Pro) + Rot, and (8) the levodopa/curcumin co-loaded lf-protocells group: (40 $\mu \mathrm{M}$ levodopa 
$+2 \mu \mathrm{M}$ curcumin)-loaded lf-protocells (L/C-Lf-Pro) + Rot. The molar concentration of levodopa and curcumin loaded in C-Lf-Pro, L-Lf-Pro or L/C-Lf-Pro group were equivalent to corresponding free drug group.

\subsection{Animals}

The male C57BL/6 mice (10 weeks old) and male BALB/c nude mice (initial weight of $18-20$ g) provided by SPF (Beijing) Biotechnology Co., Ltd were kept under specific-pathogen-free condition. All animal experimental procedures were performed in accordance with guidelines approved by the ethics committee of Chinese Academy of Medical Science \& Peking Union Medical College. Mice had free access to standard chow diet and water and were maintained in plastic cages filled with hardwood chips in a temperature-controlled room $\left(24{ }^{\circ} \mathrm{C}\right)$ on a $12: 12 \mathrm{~h}$ light/dark cycle for 7 days prior to the experiments.

\subsection{Synthesis of MSNs}

Various mesoporous silica nanoparticles were prepared according to the literature with little modification [32]. In brief, $0.437 \mathrm{~g}$ of $\mathrm{CTAB}$ and $0.472 \mathrm{~g}$ of Brij58 were dissolved in $100 \mathrm{~mL}$ of $0.1 \mathrm{M}$ phosphate buffer solution $(\mathrm{pH}=7.4)$. With vigorous stirring for $20 \mathrm{~min}$ at $60{ }^{\circ} \mathrm{C}, 2.14 \mathrm{ml}$ of TEOS was added to the solution at a rate of approximately 30 drops per minute, and the stirring process at $60{ }^{\circ} \mathrm{C}$ was continued for another $8 \mathrm{~h}$. In order to enlarge the pore size to increase adsorption, $2 \mathrm{ml}$ of TMB was added after the addition of TEOS for $40 \mathrm{~min}$. Then, the stirring process at $60{ }^{\circ} \mathrm{C}$ 
was continued for another $8 \mathrm{~h}$. To distinguish, we called the product synthesized without adding TMB as small pore MSNs (sp-MSNs), while synthesized with adding TMB as MSNs. The resulting white precipitate of sp-MSNs or MSNs was purified to remove remaining surfactant by centrifugation and washed with ethanol and water three times each. To remove the organic template, the sediment was refluxed for $24 \mathrm{~h}$ at $78{ }^{\circ} \mathrm{C}$ in acidic ethanolic solution (ethanol: hydrochloric acid $(\mathrm{HCl})=60: 1, \mathrm{v} / \mathrm{v}$ ). And the resulting white precipitate was purified by centrifugation and washed with ethanol for three times.

\subsection{Characterization of sp-MSNs and MSNs}

For measuring particle size distribution such as mean particle size as well as PDI, sp-MSNs and MSNs were diluted to an appropriate volume with distilled water and measured by the dynamic light scattering (DLS) method (Malvern Instruments, UK) at room temperature. The nitrogen adsorption/desorption analysis was performed using an adsorption analyzer (ASAP 2460, Micromeritics, USA). According to the adsorption data, the Brunauer-Emmett-Teller (BET) and Barrett-Joyner-Halenda (BJH) models were used to calculate the specific surface areas and the pore size of sp-MSNs and MSNs. Morphology of sp-MSNs and MSNs were observed via transmission electron microscopy (TEM, JEM-100CX II, JEOL, Japan).

\subsection{Preparation of lf-protocells and drug loading}

Refer to Huan Meng's synthetic method of protocells, the lipid bilayer (LB) 
coating with MSNs were achieved as follow [33]. The coating lipid bilayer consisted of a DPPC/Chol/DSPE-PEG 2000 mixture, the molar ratio of which was 75:20:5. These three excipients were dissolved in chloroform and placed into a round-bottom flask, the resulting lipid solution was dried to a film by a rotary vacuum evaporator (RV10 digital, IKA, Germany) for $15 \mathrm{~min}$ at room temperature. Following the addition of 2 $\mathrm{mL}$ MSNs suspension to the as-prepared coating lipid film at a ratio of $1: 1$ (w/w dry weight), probe sonication was used for 15 min with 15/15 s on/off working cycle at a power output of $50 \mathrm{~W}$. Since the resulting suspension contained protocells, liposomes and others, the protocells were separated by centrifugation at $12000 \mathrm{rpm}$ for $10 \mathrm{~min}$, followed by washing three times in saline.

Lactoferrin modified protocells (denoted as lf-protocells) were prepared according to a previously described method with slight modifications [28]. Lactoferrin (Lf) was dissolved in borate-EDTA buffer ( $\mathrm{pH}$ 8.0) containing Traut's reagent for $2 \mathrm{~h}$ at $4^{\circ} \mathrm{C}$. The obtained Lf-SH was mixed with DSPE-PEG $2000-\mathrm{MAL}$ in PBS ( $\mathrm{pH} 7.0$ ) for $24 \mathrm{~h}$ under constant shaking in the dark at $4^{\circ} \mathrm{C}$. Thereafter, the product was dialyzed and freeze-dried to acquire the conjugate DSPE-PEG $\mathrm{C}_{2000}-\mathrm{Lf}$ in powder form. The coating lipid bilayer consisted of a DPPC/Chol/DSPE-PEG $2000 / D_{S P E}-\mathrm{PEG}_{2000}$-Lf mixture, the molar ratio of which was 75:20:4:1. Finally, 1f-protocells were obtained according to the above preparation method of protocells.

To obtain levodopa-soaked MSNs, levodopa was dissolved in $\mathrm{HCl}(\mathrm{pH} 1.0)$ with a concentration of $10 \mathrm{mg} / \mathrm{mL}$, then $10 \mathrm{mg}$ of MSNs were soaked in $2 \mathrm{~mL}$ of levodopa 
solution for $48 \mathrm{~h}$ under light-sealed condition at room temperature.

To obtain curcumin loaded lipid film, $200 \mu \mathrm{g}$ curcumin was added to the mixed lipid solution followed by rotary evaporation drying to form the lipid film containing curcumin.

To achieve co-loading of levodopa/curcumin, the levodopa-soaked MSNs suspension was added to the lipid film containing curcumin, followed by rehydration, sonication, centrifugation, and washing, similar to preparation method of protocells described above. The Fig. 1A depicted the preparation procedure for lf-protocells as a carrier for delivery of a combination of levodopa and curcumin.

\subsection{Characterization of lf-protocells}

The conjugate DSPE-PEG 2000 -Lf was identified by ${ }^{1} \mathrm{H}-\mathrm{NMR}$ spectroscopy. DSPE-PEG ${ }_{2000}-\mathrm{MAL}$ and DSPE-PEG ${ }_{2000}-\mathrm{Lf}$ were dissolved in $\mathrm{D}_{2} \mathrm{O}$ and analyzed in a ${ }^{1}$ H-NMR spectrometer (Bruker, Switzerland).

For measuring particle size distribution such as mean particle size and PDI of various lf-protocells (unloaded and drugs-loaded), nanoparticles were diluted to an appropriate volume with distilled water and measured by the DLS method (Malvern Instruments, UK) at room temperature. Unloaded and drugs-loaded lf-protocells were characterized for morphology using a TEM (JEM-100CX II, JEOL, Japan). A minimum of three images for each sample was captured.

2.8 Encapsulation efficiency and drug loading content of lf-protocells 
drug-loaded lf-protocells were determined by a subtraction method as the following equations:

$$
\begin{aligned}
& \mathrm{EE}(\%)=\frac{\text { (total mass of drug in loading) }-(\text { the mass of non-encapsulated drug) }}{\text { total mass of drug in loading }} \times 100 \\
& \operatorname{DLC}(\%)=\frac{\text { (total mass of drug in loading) }-(\text { the mass of non-encapsulated drug) }}{\text { total mass of MSNs }} \times 100
\end{aligned}
$$

The concentration of levodopa was determined by high-performance liquid

269 chromatography (HPLC) with the mobile phase composed of tetrahydrofuran and $0.1 \%$ TFA $(3: 97, \mathrm{v} / \mathrm{v})$, and UV detection operated at $280 \mathrm{~nm}$; while the concentration of curcumin was determined by HPLC with the mobile phase composed of methanol and $4 \%$ acetic acid $(75: 25, \mathrm{v} / \mathrm{v})$, and UV detection operated at $430 \mathrm{~nm}$.

2.9 Hemolysis assay of MSNs and lf-protocells

Hemolysis assays were used to assess the safety of MSNs and lf-protocells for in vivo applications. After collection of beagle dog blood samples, red blood cells (RBCs) were collected by centrifugation at $1000 \mathrm{rpm}$ for $10 \mathrm{~min}$. After discarding the supernatant and washing three times with sterile isotonic saline, the RBCs suspension was diluted to a concentration of $2 \%(\mathrm{v}: \mathrm{v})$. Subsequently, $0.4 \mathrm{~mL}$ of $2 \%$ RBCs suspensions were mixed with $0.8 \mathrm{~mL}$ of MSNs (or lf-protocells) suspensions in saline at $50,100,200,400,600,800$, and $1000 \mu \mathrm{g} / \mathrm{mL}$ concentration. The hemolysis of RBCs in saline and $5 \%$ Triton $\mathrm{X}-100$ served as negative and positive control, respectively. The mixtures were incubated at $37{ }^{\circ} \mathrm{C}$ for $1 \mathrm{~h}$ and then centrifuged at $1000 \mathrm{rpm}$ for $3 \mathrm{~min}$. The supernatants were measured at $540 \mathrm{~nm}$ using a microplate 
reader (BioTek Synergy H1, USA). The following formula was used to calculate the hemolysis percentage:

Hemolysis percentage $(\%)=\frac{(\text { absorbance of the sample }- \text { absorbance of the negative control })}{(\text { absorbance of the positive control - absorbance of the negative control })} \times 100$

2.10 In Vitro cell proliferation and viability by MTT assay

Cellular toxicity of nanoparticles was determined using a colorimetric assay with MTT. SH-SY5Y cells were seeded in 96-well plates $\left(1 \times 10^{4}\right.$ per well $)$ and incubated for $24 \mathrm{~h}$. Subsequently, cells were exposed to various concentrations of MSNs or lf-protocells for an additional period of $24 \mathrm{~h}$. Cell viability assays were then determined by MTT.

Protection effects of free drug and drug-loaded lf-protocells against rotenone-induced cytotoxicity were also determined using a colorimetric assay with MTT. SH-SY5Y cells were cultured in 96-well plates $\left(1 \times 10^{4}\right.$ per well $)$ for $24 \mathrm{~h}$.

To determine the toxicity of rotenone, SH-SY5Y cells were incubated with different concentrations of rotenone $(0.5,1,2,4,10$ and $20 \mu \mathrm{M})$ for $24 \mathrm{~h}$. After co-incubated for $24 \mathrm{~h}$, cell viability assays were then determined by MTT.

To determine the neuroprotective effects of free drugs (levodopa and curcumin), SH-SY5Y cells were pretreated with free drug. A series of concentrations of curcumin were $1,2,4 \mu \mathrm{M}$ and the concentrations of levodopa were $20,40 \mu \mathrm{M}$. Then these cells were treated with $4 \mu \mathrm{M}$ rotenone. After co-incubated for $24 \mathrm{~h}$, cell viability assays were then determined by MTT.

To determine the neuroprotective effects of drug-loaded and unloaded 
1f-protocells, SH-SY5Y cells were pretreated with these lf-protocells. Then the cells were treated with $4 \mu \mathrm{M}$ rotenone. After co-incubated for $24 \mathrm{~h}$, cell viability assays were then determined by MTT.

For the MTT assay, each well was treated with $100 \mu 1$ of MTT-labeling reagent $(0.5 \mathrm{mg} / \mathrm{ml})$, and the plate was incubated for an additional $3 \mathrm{~h}$. The resulting formazan crystals were dissolved with $150 \mu$ of dimethyl sulfoxide, and MTT reductions were detected at $595 \mathrm{~nm}$ by the microplate reader (BioTek Synergy H1, USA).

\subsection{Oxidative stress detection}

2.11.1 Measurement of intracellular reactive oxygen species (ROS)

In order to determine the cellular ROS level, Reactive Oxygen Species Assay Kit was used. Intracellular ROS level was measured by 2', 7'-dichlorofluorescein diacetate (DCFH), which can be oxidized into fluorescent DCF. SH-SY5Y cells (about $1 \times 10^{5}$ cells/well in 6-well plates) were cultured for $24 \mathrm{~h}$. After treatment as described in part 2.2 above for $24 \mathrm{~h}$, cells were washed twice with PBS and then incubated with $4 \mu \mathrm{M}$ DCFH-DA in the dark for $20 \mathrm{~min}$. Cells were washed twice with PBS and harvested in trypsinization. All samples were measured via FACS calibur flow cytometer (BD, USA). Fluorescent measurements were done with excitation and emission wavelengths set at $488 \mathrm{~nm}$ and $525 \mathrm{~nm}$, respectively. The experiment was repeated in triplicate.

\subsubsection{Measurement of intracellular superoxide dismutase (SOD) activity}


WST-8. SH-SY5Y cells were seeded in a 6 -well plate at $1 \times 10^{5}$ cells/well and treated

331 for $24 \mathrm{~h}$ as described in part 2.2 above. Cells were harvested and washed twice with PBS. Then, the SOD activity of the sample was determined according to the manufacturer's instructions, and the absorbance value was read at $450 \mathrm{~nm}$ by the microplate reader (BioTek Synergy H1, USA). The protein content was determined using the BCA protein assay. Each experiment was performed in triplicate.

\subsubsection{Determination of cellular total glutathione (GSH) level}

The total GSH level was quantified by GSH and GSSG Assay Kit. SH-SY5Y cells were seeded in a 6 -well plate at $1 \times 10^{5}$ cells/well and treated as described in part 2.2 above. After $24 \mathrm{~h}$ incubation, cells were collected and washed twice with PBS. was removed, then $1 \mathrm{~mL}$ FBS-free medium with $1 \mu \mathrm{M}$ Rh123 was added in, the 
flow cytometer (BD Biosciences, USA).

To visualize the mitochondrial function of cultured cells, the medium was removed after $24 \mathrm{~h}$ incubation. Each sample was incubated with $1 \mu \mathrm{M}$ Rh123 for 30 min in the dark at $37^{\circ} \mathrm{C}$. Then cells were washed twice with PBS and photographed immediately using an Ultra High Resolution Microscope (TCS SP8 STED, Leica, Germany). The results were expressed as mean Rh123 fluorescence intensity. The experiment was repeated in triplicate.

\subsection{Western blot}

SH-SY5Y cells were lysed in RIPA lysis buffer supplemented with protease inhibitors. After quantified by BCA protein assay, samples were mixed with loading buffer (containing DTT) at a ratio of $4: 1$, and then boiled for $10 \mathrm{~min}$. The resulting proteins were separated with SDS-PAGE and transferred onto a PVDF membrane. Then the membrane was sealed up in TBST with $5 \%$ skim milk at room temperate for $2 \mathrm{~h}$, and subsequently cultured with primary antibodies including anti- $\mathrm{TH}$, anti- $\alpha$-synuclein, anti-Bcl-2, anti-Bax, anti-Caspase-3, anti-PARP, anti- $\beta$-actin and anti-GAPDH at $4{ }^{\circ} \mathrm{C}$ overnight. After TBST-washing in triplicate, the membrane was incubated with secondary antibody HRP-conjugated Affinipure Goat Anti-Mouse $\operatorname{IgG}(\mathrm{H}+\mathrm{L})$ and Anti-Rabbit $\operatorname{IgG}(\mathrm{H}+\mathrm{L})$ for $1 \mathrm{~h}$ at room temperature. Protein bands were visualized using enhanced chemiluminescence. The results were analyzed by Image J. GAPDH and $\beta$-actin were included as internal controls. The experiment was repeated in triplicate. 
2.14 Cellular uptake

To investigate the uptake behavior of protocells (unmodified with Lf) and lf-protocells, fluorescence-labeled protocells and lf-protocells (denoted as RBITC/Calcein-protocells and RBITC/Calcein-lf-protocells, respectively) with RBITC-conjugated and Calcein-soaked MSNs as the inner cores were prepared.

For RBITC-conjugation, $8.6 \mathrm{mg}$ RBITC and $200 \mu \mathrm{L}$ APTES were dissolved in $2 \mathrm{~mL}$ absolute ethanol. The solution reacted for $24 \mathrm{~h}$ in the dark under stirring. RBITC-conjugated MSNs were prepared as follow: CTAB, Brij58 and TEOS were added as described above. After that, $200 \mu \mathrm{L}$ of the reacted solution was added. The following procedures were the same as the synthetic method of MSNs. For Calcein-soak, Calcein was soaked following a similar approach of levodopa-soaked MSNs, as described above. RBITC/Calcein-protocells and RBITC/Calcein-lf-protocells were prepared refer to synthetic method of protocells as described above.

For cellular uptake assay, bEnd.3 cells were seeded on glass bottom dishes at a density of $5 \times 10^{4}$ cells/mL per chamber and incubated for $24 \mathrm{~h}$ under an atmosphere of $5 \% \mathrm{CO}_{2}$ at $37 \quad{ }^{\circ} \mathrm{C}$. The cells were then treated with $200 \mu \mathrm{g} / \mathrm{mL}$ RBITC/Calcein-protocells or RBITC/Calcein-lf-protocells for $4 \mathrm{~h}$. After washing three times with PBS, the cells were treated with $4 \%$ paraformaldehyde for $30 \mathrm{~min}$ at $4{ }^{\circ} \mathrm{C}$ and the nucleus were subsequently labeled with DAPI. After that, the dishes were visualized under Ultra High Resolution Microscope (TCS SP8 STED, Leica, 
Germany). The fluorescence intensity of RBITC was determined with an excitation wavelength of $540 \mathrm{~nm}$ and an emission wavelength of $625 \mathrm{~nm}$. The fluorescence intensity of Calcein was determined with an excitation wavelength of $496 \mathrm{~nm}$ and an emission wavelength of $515 \mathrm{~nm}$.

\subsection{In Vivo Study}

\subsubsection{Biodistribution}

To investigate the biodistribution behavior of lf-protocells in mouse, fluorescence -labeled lf-protocells denoted as Cy5-lf-protocells were prepared using Cy5-conjugated MSNs as the inner core. For synthesis of Cy5-conjugated MSNs, 2.5 mg Cy5-SE and $200 \mu \mathrm{L}$ APTES were dissolved in 2mL DMSO. The solution reacted for $24 \mathrm{~h}$ in the dark under stirring. Cy5-conjugated MSNs were prepared as follow: CTAB, Brij58 and TEOS were added as described above. After that, $200 \mu \mathrm{L}$ of the reacted solution was added. The following procedures were the same as the synthetic method of MSNs.

The male BALB/c nude mice $(\mathrm{n}=3)$ were given $200 \mu \mathrm{L}$ of $5 \mathrm{mg} / \mathrm{ml}$ Cy5-labeled lf-protocells via intraperitoneal injection. Then, the mice were anesthetized by isoflurane inhalation and scanned in an IVIS Spectrum CT system (Maestro2, CRI, USA) at various time points. The images of the mice were collected at an excitation wavelength of $649 \mathrm{~nm}$ and an emission wavelength of $670 \mathrm{~nm}$.

\subsubsection{Open-Field Test}


Male C57BL/6 mice were randomly assigned to six groups $(\mathrm{n}=8 /$ group $)$ :

One group were treated with saline only (Control). PD model mice were induced by MPTP, received intraperitoneal (i.p.) injections of MPTP $(20 \mathrm{mg} / \mathrm{kg} / \mathrm{day})$ for 5 days (MPTP). Various therapeutic groups were treated with $20 \mathrm{mg} / \mathrm{kg}$ free levodopa (L), $20 \mathrm{mg} / \mathrm{kg}$ free levodopa $+2 \mathrm{mg} / \mathrm{kg}$ free curcumin (free $\mathrm{L} / \mathrm{C}$ ), levodopa/curcumin co-loaded protocells (L/C-Pro, encapsulated with $20 \mathrm{mg}$ levodopa and $2 \mathrm{mg}$ curcumin), and levodopa/curcumin co-loaded lf-protocells (L/C-Lf-Pro, encapsulated with $20 \mathrm{mg}$ levodopa and $2 \mathrm{mg}$ curcumin) respectively using i.p. injections at $1 \mathrm{~h}$ before MPTP treatment for 5 days [34].

To evaluate the exploratory and locomotor activities by Open-Field Test, each mouse was placed into the center of a square open-field box $(24 \mathrm{~cm} * 24 \mathrm{~cm} * 10 \mathrm{~cm}$, divided into sixteen squares with grids, made of transparent PVC) and acclimated for $10 \mathrm{~min}$. The motor function of each mouse was evaluated by counting the number of line crossings during $5 \mathrm{~min}$ in the square open-field box, persist for five days [34].

\subsection{Data processing}

All data were presented as mean \pm SEM (standard error of mean) of triplicates measurement. All statistical analysis was done in Graph Pad Prism software. Statistical differences between groups were evaluated using a two-tailed Student's t test and differences with a $\mathrm{P}<0.05$ were considered significant.

\section{Results and discussion}




\subsection{Characterization of sp-MSNs and MSNs}

MSNs nanoparticles were synthesized by the sol-gel phase transition method, in which TEOS was used as a silica source, CTAB and Brij-58 as the structure-directing agents [32]. With a slight modification, TMB was used for enlarging pores to provide a higher loading capacity [35].

The DLS test indicated that the prepared MSNs had outer diameter about $90 \mathrm{~nm}$, similar like the sp-MSNs without TMB modification (Tab S1). TEM images showed that, the prepared MSNs retained the high monodispersity and spherical morphology, and meanwhile possessed larger porosity compared with the sp-MSNs without TMB modification (Fig. S1). According to nitrogen adsorption data, with the enlarging pores modification, the BET surface area, pore volume, and pore size were changed from $743.78 \mathrm{~m}^{2} / \mathrm{g}, 1.01 \mathrm{~cm}^{3} / \mathrm{g}$, and $5.44 \mathrm{~nm}$ to $572.99 \mathrm{~m}^{2} / \mathrm{g}, 1.15 \mathrm{~cm}^{3} / \mathrm{g}$, and $8.00 \mathrm{~nm}$ respectively (Fig. S2, Tab. S2).

3.2 Characterization of lf-protocells and drug loading

As for ${ }^{1} \mathrm{H}-\mathrm{NMR}$ spectra analysis, the solvent peak of $\mathrm{D}_{2} \mathrm{O}$ at $\delta 4.71 \mathrm{ppm}$ was used as the reference (Fig. 1B). The ${ }^{1} \mathrm{H}-\mathrm{NMR}$ spectrum of DSPE-PEG $2000-\mathrm{MAL}$ clearly showed characteristic peak of MAL group at $\delta 6.84$ ppm (Fig. 1B), while the disappearance of MAL peak in the ${ }^{1} \mathrm{H}-\mathrm{NMR}$ spectrum of DSPE-PEG 2000 -Lf (Fig. 1B) matched well with the fact that the MAL group had been reacted with the thiol group of Lf-SH. The ${ }^{1} \mathrm{H}-\mathrm{NMR}$ spectrum demonstrated the formation of DSPE-PEG $2000-\mathrm{Lf}$.

The DLS test indicated that lf-protocells were larger than MSNs, up to $177 \mathrm{~nm}$ in 
diameter. The presence of the coating lipid bilayer was confirmed by TEM. As can be seen, the lf-protocells appeared to be surrounded by a ring, and the appearances of the pores were relatively vague compared with TEM image of MSNs shown in Fig. S1.

The co-loading of levodopa and curcumin had little influence on the morphology of 1f-protocells (Fig. 1C).

And the results of DLC and EE of various lf-protocells were shown in Tab. 1. L-Lf-Pro yielded a levodopa DLC of 21.59 wt \% (drug: MSNs) and a levodopa EE of $10.80 \mathrm{wt} \%$, which were quite similar to that of binary-drug loaded lf-protocells (DLC of $20.28 \mathrm{wt} \%$ and EE of $10.14 \mathrm{wt} \%$ for levodopa).

As previously mentioned, hydrophobic drug, such as curcumin, could be loaded in the outer membrane LB and have a synergistic effect with levodopa to treat Parkinson's disease in theory.

HPLC analysis indicated that the C-Lf-Pro yielded a curcumin DLC of 1.93 wt \% (drug: MSNs) and a curcumin EE of $96.57 \mathrm{wt} \%$, which were also quite similar to that of binary-drug loaded lf-protocells (DLC of $1.97 \mathrm{wt} \%$ and EE of $98.32 \mathrm{wt} \%$ for curcumin).

The co-loading of levodopa and curcumin showed a similar DLC and EE value to that of single-drug loaded lf-protocells, which was attributable to the possible deep immersion of levodopa in the MSNs core and the immersion of curcumin in the lipid bilayer.

In addition, the in vitro release profiles of levodopa and curcumin from binary-drug loaded lf-protocells in neutral or acidic medium were measured using 
HPLC (Fig. S3). Neutral medium of $\mathrm{pH} 7.4$ simulated body fluid, and acidic medium of $\mathrm{pH} 5.0$ simulated endosomal/lysosomal environment. The results suggested that no levodopa and only 59.2\% curcumin was released from lf-protocells for $48 \mathrm{~h}$ in $\mathrm{pH} 7.4$ solution at $37^{\circ} \mathrm{C}$. When the $\mathrm{pH}$ is decreased to acidic condition (pH 5.0), lf-protocells released $100.4 \%$ of their curcumin and $72.6 \%$ of their levodopa within 48 hours. Therefore, we speculated that the release of levodopa was hindered by the inclusion of lipid bilayer outside the inner core, which would greatly reduce its leakage in the circulatory system. However, the drug release from lf-protocells in acidic conditions is initiated by LB destabilization.

Fig. 1 Preparation and characterization of If-protocells of If-protocells.

(A) Scheme of the preparation of lf-protocells as a carrier for co-delivery of levodopa and curcumin. (B) ${ }^{1} \mathrm{H}-\mathrm{NMR}$ spectra of DSPE-PEG $2000-\mathrm{MAL}$ (top) and DSPE-PEG $2000-\mathrm{Lf}$ (bottom) ( $\mathrm{D}_{2} \mathrm{O}$ as the solvent). (C) TEM images of various lf-protocells. (1) unloaded lf-protocells; (2) levodopa loaded lf-protocells; (3) curcumin loaded lf-protocells; (4) binary-drug loaded lf-protocells.

Tab 1. Physicochemical characteristics of various Lf-Protocells (mean $\pm \mathrm{SD} ; \mathbf{n}=\mathbf{3}$ ).

\begin{tabular}{|c|c|c|c|c|}
\hline & unloaded & levodopa & curcumin & binary-drug loaded \\
\hline & Lf-Protocells & loaded & loaded & Lf-Protocells \\
\hline & & Lf-Protocells & Lf-Protocells & levodopa \\
\hline Particle size & $176.2 \pm 1.4$ & $226.8 \pm 5.4$ & $207.6 \pm 0.8$ & $219.3 \pm 7.2$ \\
\hline
\end{tabular}


$(\mathrm{nm})$

$\begin{array}{cccccc}\text { PDI } & 0.176 \pm 0.002 & 0.154 \pm 0.005 & 0.205 \pm 0.007 & 0.149 \pm 0.005 \\ \text { EE (\%) } & - & 9.87 \pm 0.38 & 86.67 \pm 0.12 & 10.72 \pm 0.04 & 88.31 \pm 0.02 \\ & - & & & & \\ \text { DLC (\%) } & - & 20.89 \pm 0.34 & 1.73 \pm 0.02 & 21.44 \pm 0.09 & 1.89 \pm 0.01\end{array}$

501

3.3 Biocompatibility and cytotoxicity of MSNs and 1f-protocells

503 Hemolysis and cell viability assays were performed to investigate

504 biocompatibility and cytotoxicity of MSNs (as the inner core of 1f-protocells) and 505 1f-protocells.

The hemocompatibility of MSNs, protocells and lf-protocells was evaluated by

507 hemolysis assay at the concentrations ranging from 50 to $1000 \mu \mathrm{g} / \mathrm{mL}$. The hemolytic

508 activity of MSNs, protocells and lf-protocell on RBCs was observed using digital

509 photography and calculated by the formula of hemolysis percentage as described

510 above (Fig. S4). Visual inspection of the hemolysis experiments showed a clear color

511 differential between samples with varying degrees of hemolysis. Uncoated MSNs

512 showed high hemolytic activity, roughly $40 \%$ of the positive hemolysis control of

$513 \mathrm{RBCs}$ when the concentration exceeded $400 \mu \mathrm{g} / \mathrm{mL}$. In contrast, with the lipid bilayer

514 (LB) coating, protocells showed the hemolytic activity below $5 \%$ of the positive

515 hemolysis control of RBCs, even at concentrations greater than $800 \mu \mathrm{g} / \mathrm{mL}$. In

516 addition, the hemolysis ratio of lf-protocells with a concentration of $1000 \mu \mathrm{g} / \mathrm{mL}$ was

517 still less than $5 \%$ compared with the positive control group. Studies on other particle

518 systems have determined that increasing the positive charge density of the particle 
leads to a decrease in hemolysis. When acidic silanols on MSNs have been masked by the lipid bilayer, no hemolysis is observed [36]. This result demonstrated that the LB coating on the surface of MSNs could improve markedly the biocompatibility of silica, and modification of Lf ligand could not increase hemolytic activity. These results suggested that the LB coating could reduce dramatically the hemolysis percentage, and improve significantly the biocompatibility of lf-protocells.

The in vitro cell viability assays were performed by MTT to evaluate the toxicity of the MSNs and lf-protocells on SH-SY5Y cells. As shown in Fig. S5, over $90 \%$ cell viability was achieved after incubating SH-SY5Y cells with $200 \mu \mathrm{g} / \mathrm{mL}$ of MSNs and lf-protocells respectively for $24 \mathrm{~h}$, while over $85 \%$ cell viability was achieved after incubating SH-SY5Y cells with $500 \mu \mathrm{g} / \mathrm{mL}$ of particles for $24 \mathrm{~h}$. The results suggested that MSNs and 1f-protocells possessed low cytotoxicity while the concentration was below $40 \mu \mathrm{g} / \mathrm{mL}$.

\subsection{Cytotoxicity of curcumin, levodopa and rotenone alone on SH-SY5Y cells}

The cytotoxicity of curcumin alone, levodopa alone and rotenone alone to SH-SY5Y dopaminergic cell line was evaluated. Cells were treated with various concentrations of curcumin $(1,2,4 \mu \mathrm{M})$, levodopa $(20,40 \mu \mathrm{M})$ and rotenone $(0.5,1,2$, $4,10,20 \mu \mathrm{M})$ respectively for $24 \mathrm{~h}$, and the cell viability assays were determined by MTT. As shown in Fig. $2 \mathrm{C}$-D, curcumin alone below $4 \mu \mathrm{M}$ did not affect cell viability, while levodopa alone did not affect cell viability at the concentrations of 40 $\mu \mathrm{M}$ or lower. 
542 SH-SY5Y cells were spindle or polygonal and had mesh connection with

543 circumambient cell. After $24 \mathrm{~h}$ treatment, with the increase of concentration, rotenone

544 induced marked cell shrinkage, disappeared connections and loss of whole cells. The

545 above observations indicated that rotenone created the PD cell model successfully and

546 had obvious toxic effect on cellular morphology. As previously reported [37], a

547 significant dose-dependent reduction of rotenone-induced cell viability could be

548 observed. Due to the approximate median lethal dose (LD50) observed at $4 \mu \mathrm{M}$.

549 To investigate the effects of rotenone induction on SH-SY5Y cells, western blot 550 analysis on several important apoptosis mediated signaling pathway proteins was

551 performed. The expression levels of pro-apoptotic factors PARP, Caspase-3, Cleaved

552 Caspase-3, Bax and anti-apoptotic factors Bcl-2 were detected in $10 \mu \mathrm{M}$ Rot-induced

553 SH-SY5Y cells after $24 \mathrm{~h}$ cultivation. Compared with blank group, rotenone 554 significantly increased PARP, Cleaved Caspase-3 and Bax levels and cut down 555 Caspase-3 and Bcl-2 levels, which indicated promotion effect on apoptosis in a 556 dose-dependent manner (Fig. S6A).

557 Since the typical pathological changes of PD include the deformation of 558 dopaminergic neurons, the aggregation of intracellular $\alpha$-synaptic nuclear ( $\alpha$-syn) 559 protein and the decrease of TH expression, we measured the expression of $\alpha$-syn and TH in Rot-induced SH-SY5Y cells. 
563

decreased the level of $\mathrm{TH}$ and increased the level of $\alpha$-syn in a dose-dependent manner.

\subsection{Protection of curcumin and levodopa against rotenone-induced cytotoxicity}

The cytoprotective effect of curcumin or levodopa against rotenone-induced SH-SY5Y cytotoxicity was determined by pre-treating cells with different concentrations of curcumin or levodopa for $60 \mathrm{~min}$ prior to rotenone exposure. Pretreatment with curcumin alone at $1,2,4 \mu \mathrm{M}$ for $60 \mathrm{~min}$ prior to rotenone exposure significantly increased the cell viability to $79.2 \pm 4.4,82.6 \pm 5.6,76.9 \pm 4.4 \%$ of control, respectively, and pretreatment with levodopa alone at $20,40 \mu \mathrm{M}$ for $60 \mathrm{~min}$ prior to rotenone exposure also immensely enhanced the cell viability to $80.2 \pm 4.5$, $81.0 \pm 4.7 \%$ of control, respectively. While the cytoprotective effect of curcumin $\&$ levodopa combination against rotenone resulted in dramatic raise in SH-SY5Y cell viability compared with single drug treatment as shown in Fig. 2E-F. Particularly, curcumin and levodopa exerted the highest cytoprotective effect at $2 \mu \mathrm{M}$ and $40 \mu \mathrm{M}$ respectively. Therefore, curcumin of $2 \mu \mathrm{M}$ and levodopa of $40 \mu \mathrm{M}$ were chosen for the subsequent experiments. The concentration of levodopa and curcumin loaded in C-Lf-Pro, L-Lf-Pro or L/C-Lf-Pro were equivalent to corresponding free drug, respectively. 
$\mu \mathrm{M} \operatorname{Rot}(2) 1 \mu \mathrm{M} \operatorname{Rot}(3) 2 \mu \mathrm{M} \operatorname{Rot}(4) 4 \mu \mathrm{M} \operatorname{Rot}(5) 10 \mu \mathrm{M} \operatorname{Rot}(6) 20 \mu \mathrm{M}$ Rot; (B) Effect of

gradient concentration of Rot on SH-SY5Y cells viability. (mean $\pm \mathrm{SD} ; \mathrm{n}=6$. $* * * \mathrm{P}<0.001$

compared with the control group.) (C-D) Effects of gradient concentration of curcumin or

levodopa on SH-SY5Y cells viability, as well as protection against toxicity induced by $4 \mu \mathrm{M}$ Rot.

(mean $\pm \mathrm{SD} ; \mathrm{n}=6 . * * * \mathrm{P}<0.001$ compared with the $4 \mu \mathrm{M}$ Rot group.) (E-F) Protection of various

combinations of gradient concentration of levodopa and curcumin against toxicity induced by 4

$\mu \mathrm{M}$ Rot. (mean $\pm \mathrm{SD} ; \mathrm{n}=6$. ${ }^{* * *} \mathrm{P}<0.001$ compared with the $4 \mu \mathrm{M}$ Rot group. \# $\mathrm{P}<0.05$, \#\# $\mathrm{P}<$

0.01 compared between two groups.) Cells were exposed for $24 \mathrm{~h}$ and cell viability was determined by MTT assay. Rot: rotenone; C: curcumin; L: levodopa.

3.6 Protection of binary-drug loaded lf-protocells against rotenone-induced cytotoxicity

As shown in Fig. S7, exposure of rotenone $(4 \mu \mathrm{M})$ led to a dramatic decrease in SH-SY5Y cell viability compared with that in control group. We found that, the pretreatment of unloaded lf-protocells or levodopa loaded lf-protocells, could not increase cell viability. However, curcumin loaded lf-protocells and binary-drug loaded lf-protocells significantly decreased rotenone induced cytotoxicity $(\mathrm{P}<0.01)$. Additionally, treatment with binary-drug loaded lf-protocells markedly reduced rotenone-induced cytotoxicity compared with levodopa loaded lf-protocells $(\mathrm{P}<0.05)$. In brief, our study showed that binary-drug loaded lf-protocells exerted a significant 
3.7 Lf-protocells treatment ameliorated oxidative stress in SH-SY5Y cells induced by rotenone

3.7.1 Lf-protocells protected against rotenone-induced reactive oxygen species (ROS) production.

DCFH-DA, a specific ROS fluorescence probe, was used to analyze whether levodopa and curcumin could inhibit rotenone-induced intracellular ROS generation. The levels of ROS production were determined by the intensity of DCF fluorescence within cells. As shown in Fig. 3A, exposure of rotenone $(4 \mu \mathrm{M})$ led to a dramatic increase of fluorescence intensity in SH-SY5Y cells compared with that in control group. After the pretreatment of single drug alone, neither curcumin nor levodopa, could decrease the high level of intracellular ROS induced by rotenone. However, co-treatment of curcumin and levodopa (free L/C), as well as treatment of L/C-Lf-Pro, significantly decreased rotenone-induced ROS production $(\mathrm{P}<0.05, \mathrm{P}<0.001$, respectively). Interestingly, treatment with L/C-Lf-Pro markedly scavenged rotenone-induced ROS compared with free L/C group $(\mathrm{P}<0.05)$, L-Lf-Pro group $(\mathrm{P}<$ 0.001), and C-Lf-Pro group ( $\mathrm{P}<0.01)$, respectively. In brief, our study showed that L/C-lf-protocells exerted significant effect on reduction of intracellular ROS level in SH-SY5Y cells activated by rotenone.

\subsubsection{Lf-protocells improved the SOD activity.}

Superoxide dismutase (SOD), as an anti-oxidative defense enzyme, plays a crucial role in maintaining cellular redox homeostasis [38]. Thus, we next determined 
629

630

631

632

633

634

635

636

637

638

639

640

641

642

643

644

645

646

647

648

649

650

the effect of lf-protocells on the enzyme activities of SOD. As shown in Fig. 3B, rotenone $(4 \mu \mathrm{M})$ resulted in a marked decrease in SOD activity of SH-SY5Y cells $(\mathrm{P}<$ 0.01). Oxidative stress induced by rotenone was confirmed by the reduced expression of SOD. While curcumin \& levodopa co-treatment in the form of free drugs (free L/C) and lf-protocells (L/C-Lf-Pro) significantly improved the enzyme activities of SOD reduced by rotenone $(\mathrm{P}<0.01)$, and both of them were superior to corresponding single drug treatment $(\mathrm{P}<0.05)$. In addition, pretreatment with L/C-Lf-Pro markedly increased the activity of SOD to $93.5 \%$, the highest among all groups, even though there was no statistical difference compared with free L/C group. In short, our study indicated that L/C-Lf-Pro could efficiently recover the SOD activity impaired by rotenone.

3.7.3 Lf-protocells improved rotenone-induced GSH depletion.

GSH is a tripeptide nonprotein anti-oxidant and redox regulator in brain in which GSH depletion observed probably occurs via oxidative damages caused by increased ROS with significant mitochondrial damage [39]. Based on GSH neutralizing free radicals and reactive oxygen compounds in cells as described above, the assessment of intracellular GSH content should be thought about in further exploration. Since lf-protocells exhibited a profound effect on scavenging Rot-induced intracellular ROS and ameliorating SOD activity, we speculated that lf-protocells could also improve Rot-induced GSH depletion in SH-SY5Y cells.

As shown in Fig. 3C, the content of total GSH decreased remarkably after 
651

652

653

654

655

656

657

658

659

660

661

662

663

664

665

666

667

668

669

670

671

672

incubation with rotenone $(4 \mu \mathrm{M})$ (compared with control group), whereas the total GSH content increased after the pretreatment with levodopa/curcumin for $24 \mathrm{~h}(\mathrm{P}<$ 0.01), as the same trend as that on the improved SOD activity. Noticeably, pretreatment with L/C-Lf-Pro attenuated Rot-induced total GSH depletion, the best among all groups, even though there was no statistical difference compared with free

L/C group. These observations implied that L/C-Lf-Pro might provide protection against Rot-induced oxidative damage in SH-SY5Y cells via regulating GSH antioxidant system to cope with oxidative stress.

The occurrence and development of PD are closely related to oxidative stress, which are involved in the process of neuronal deformation in PD. The anti-oxidative therapeutic efficacy of binary-drug loaded lf-protocells were apparently confirmed by SOD and GSH experiment in vitro. The results of SOD and GSH were in conformity with the ROS assay. These findings suggested that levodopa and curcumin co-loaded lf-protocells, a novel nanoparticle platform comprised of a Lf-modified lipid bilayer (LB) containing curcumin as its outer membrane and MSNs containing levodopa as its supporting inner core, compared with free levodopa and curcumin, could effectively enhance the level of total GSH and SOD in damaged cells, reduce the oxidative damage, and thus protect cells against oxidative stress, improve the anti-oxidative therapeutic effect for PD.

\section{Fig. 3 Lf-protocells treatment ameliorated oxidative stress in SH-SY5Y cells induced by Rot.}

(A) Treatments with lf-protocells protected against Rot-induced ROS in SH-SY5Y cells. The 
673

674

levels of intracellular ROS were quantified by fluorescence with DCF. The fluorescence data were obtained by flow cytometry. (B) Effect of lf-protocells on SOD activity reduced by Rot. SOD activity was measured and expressed as $\mathrm{U} / \mu \mathrm{g}$ protein. (C) Lf-protocells improved Rot-induced total GSH depletion in SH-SY5Y cells. mean $\pm \mathrm{SD}, \mathrm{n}=3 .{ }^{*} \mathrm{P}<0.05,{ }^{* *} \mathrm{P}<0.01,{ }^{* * *} \mathrm{P}<0.001$ compared with the $4 \mu \mathrm{mol} / \mathrm{L}$ Rot group. \# $\mathrm{P}<0.05$, \#\# $\mathrm{P}<0.01$, \#\#\# $\mathrm{P}<0.001$, compared between two groups. Rot: rotenone; C: curcumin; L: levodopa; Lf-Pro: lf-protocells.

\subsection{Lf-protocells suppressed rotenone-induced reduction of the MMP}

As the most important organelle, mitochondria play a key role not only in supplying metabolic energy to cells in the form of ATP via direct participating in a number of metabolic reaction, but also in regulating the signal transmission during the apoptosis of cells [40]. In addition, although electron transport chain in mitochondria is the main source of intracellular ROS, mitochondrial dysfunction, including the collapse of MMP, the membrane non-integrity and membrane oxidative damage and so on, can be aroused by high ROS exposure [41].

As we know, the burst of ROS and depletion of intracellular SOD and total GSH can induce the drop of MMP. The collapse of MMP, in turn, can also stimulate ROS generating excessively and the level of SOD and total GSH declining [38]. Since lf-protocells exhibited a profound effect in ameliorating oxidative stress in SH-SY5Y cells induced by rotenone, we postulated that lf-protocells could also ameliorate Rot-induced oxidative damage to membrane and mitochondrial dysfunction.

According to the flow cytometry charts, the quantitative data were shown in the 
695

696

697

698

699

700

701

form of bar diagrams. As shown in Fig. 4A, the Rh123 fluorescence intensity of rotenone group was about half of that of control group, a remarkable decrease of fluorescence intensity was observed after treatment with rotenone $(\mathrm{P}<0.001)$. Pretreatment with free drugs (including L group, C group and free L/C group), attenuated the decrease of Rot-induced mitochondrial function as reflected by increasing the fluorescence intensity of rotenone group $(\mathrm{P}<0.05)$. In addition, pretreatment with L/C-Lf-Pro increased significantly the fluorescence intensity compared with that of free L/C group $(\mathrm{P}<0.05)$.

Furtherly, the change of Rh123 fluorescence intensity can be displayed by a visual way with the help of fluorescent confocal microscope (Fig. 4B). The green light intensity characterizing mitochondrial function weakened owing to the addition of rotenone, followed by becoming stronger after treatment with free $\mathrm{L} / \mathrm{C}$ or L/C-Lf-Pro, which were in accordance with the former results.

Our preliminary data demonstrated that L/C-Lf-Pro enabled to inhibit Rot-induced ROS accumulation. Further research revealed that L/C-Lf-Pro played a role in preventing mitochondrial oxidation due to improving the SOD activity and recovering the level of total $\mathrm{GSH}$, thereby decreasing the oxidative damage to mitochondrial membrane. Since MMP reduction could induce apoptotic signalling downstream involving cytochrome $\mathrm{C}$ release from mitochondria and subsequent caspase activation, and could also induce pro-inflammatory signaling during apoptosis [42], L/C-Lf-Pro which suppressed the reduction of MMP might reduce apoptosis. As a consequence, L/C-Lf-Pro were expected to provide protection against 
Fig. 4 Lf-protocells suppressed Rot-induced mitochondrial dysfunction in SH-SY5Y cells.

720

721

722

723

724

(A) Effect of lf-protocells on Rot-induced oxidative damage and mitochondrial dysfunction in SH-SY5Y cells. The mean fluorescence intensity (MFI) was detected in each group. (B) The Rh123 accumulation in mitochondria was detected by confocal microscope (scale bar, $25 \mu \mathrm{m}$ ). (mean $\pm \mathrm{SD} ; \mathrm{n}=3 . * \mathrm{P}<0.05,{ }^{* *} \mathrm{P}<0.01,{ }^{* * *} \mathrm{P}<0.001$ compared with the $4 \mu \mathrm{mol} / \mathrm{L}$ Rot group. \# $\mathrm{P}<0.05$, compared between two groups.) Rot: rotenone; C: curcumin; L: levodopa; Lf-Pro: lf-protocells.

3.9 Lf-protocells inhibited rotenone-induced neuronal apoptosis.

To investigate the detailed mechanisms, western blot analysis on several important apoptosis mediated signaling pathway proteins was performed. Anti-apoptotic protein Bcl-2 and pro-apoptotic protein Bax play key roles in the process of apoptosis. The Bcl-2 protein family modulates cell apoptosis by controlling the MMP. Protein Bax is the key component of apoptosis caused by mitochondrial stress and increases membrane permeability after apoptosis stimulation.

Over-expression of Bax can accelerate apoptotic death by activating caspase pathway [35]. In addition, once the mitochondrial membrane permeability suffer from damage, Bax may release from the intermembrane space to initiate caspase activation in the cytosol [43]. Protein Caspase-3 has a vital role to play executor of apoptosis and could crack poly ADP-ribose polymerase (PARP). The proenzyme form of Caspase-3 
is hydrolyzed and activated to a Cleaved Caspase-3 (17 kd subunit $+19 \mathrm{kd}$ subunit).

Moreover, PARP is one of the main shearing targets of Caspase- 3 in vivo and could be used as a marker of apoptosis.

Our results, as shown in Fig. 5A, indicated that Bcl-2 and Caspase-3 were up-regulated in both free L/C group and L/C-Lf-Pro group compared with Rot group and single drug groups. Similarly, the expression of Bax, Cleaved Caspase-3 and PARP were down-regulated in both free L/C group and L/C-Lf-Pro group compared with Rot group, and the degree of decrease was markedly enhanced in binary-drug groups compared with single-drug groups. Based on the role of apoptosis mediated signaling pathway proteins as described above, these results suggested that combination of levodopa and curcumin might partially reverse the apoptosis of cells by reducing mitochondrial damage.

Since the typical pathological changes of PD include deformation of dopaminergic neurons, aggregation of intracellular $\alpha$-synaptic nuclear ( $\alpha$-syn) protein and decrease of tyrosine hydroxylase (TH) expression. We measured the expression of PD related proteins using certain experiments.

The results of western blot on $\mathrm{TH}$ and $\alpha$-syn showed that rotenone could significantly decrease the level of TH and increase the level of $\alpha$-syn compared with blank group, while levodopa and curcumin reversed this trend (Fig. 5B). The addition of both free L/C and L/C-Lf-Pro could reduce Rot-introduced cytotoxicity as reflected by increased $\mathrm{TH}$ level and decreased $\alpha$-syn level. Interestingly, the performance of binary-drug group was better than single-drug group. In addition, since TH is the key 
enzyme in dopamine synthesis, co-delivery of levodopa and curcumin is expected to promote the dopamine synthesis by increasing TH expression. Moreover, the addition of levodopa as a dopaminergic neurotransmitter supplement would further increase dopamine levels. Therefore, the results demonstrated that levodopa and curcumin could protect dopaminergic neurons through decreasing the aggregation of $\alpha$-syn protein and increasing the activity of $\mathrm{TH}$.

Overall, these studies implied that L/C-Lf-Pro pretreatment may alleviate the apoptosis of PD cells and protect dopamine neurons through the following mechanisms: reducing the accumulation of unfolded or misfolded $\alpha$-syn proteins, enhancing the level of $\mathrm{TH}$ and transforming more levodopa into dopamine for supplementing the loss of dopamine in the brain, reducing the mitochondrial damage and dysfunction, down-regulating the expression of Bax protein and up-regulating the expression of Bcl-2, inhibiting the activation of Caspase-3 and thus exerting an anti-apoptotic ability, showing a protective effect in the degenerative changes of dopamine neurons finally.

\section{Fig. 5 The effects of levodopa/curcumin and L/C-Lf-Pro on rotenone-induced variation}

\section{in expression of apoptosis related proteins and PD related proteins through western blot} analysis.

Representative blots on the upper panels, along with bar graph summarizing group data on the bottom panels. (A) Expression of apoptosis related proteins in experimental groups. The expressions of Bax, Cleaved Caspase-3, PARP were decreased while the expressions of Bcl-2 and 
Caspase-3 were significantly increased by free L/C or L/C-Lf-Pro pretreatment compared with the $10 \mu \mathrm{mol} / \mathrm{L}$ Rot group. Pretreatment with free $\mathrm{L} / \mathrm{C}$ or L/C-Lf-Pro recovered the imbalanced expression profile of these proteins, protected SH-SY5Y cells against rotenone induced apoptosis.

(B) Expression of PD related proteins in experimental groups. Pretreatment with free $\mathrm{L} / \mathrm{C}$ or L/C-Lf-Pro reduced the accumulation of misfolded $\alpha$-syn and enhanced the level of TH, most obviously compared with other groups. (mean $\pm \mathrm{SD} ; \mathrm{n}=3$. * $\mathrm{P}<0.05, * * \mathrm{P}<0.01, * * * \mathrm{P}<0.001$ compared with the $10 \mu \mathrm{M}$ Rot group. \# $\mathrm{P}<0.05$, \#\# $\mathrm{P}<0.01$, \#\#\# $\mathrm{P}<0.001$ compared between two groups.) Rot: rotenone; C: curcumin; L: levodopa; Lf-Pro: lf-protocells.

\subsection{Cellular Uptake}

The bEnd.3 cells are the brain microvascular endothelial cells in the BBB. The BBB blocks passage of most drugs. In our study, Lf modified protocells allowed more drugs to pass through BBB by the specific receptor-mediated transport. RBITC was labeled on the inner core of protocells by chemical bond, indicating the location of protocells. Calcein is loaded in protocells by the adsorption of MSNs, indicating the location of loading drugs. The cellular uptake of protocells and their loading drugs was investigated, through labeled with RBITC and Calcein respectively.

The results of cellular uptake were shown in Fig. S8. Only the blue nucleus stained with DAPI were visible in the protocells group, and no other fluorescence was seen. While the lf-protocells group showed distinct red and green fluorescence besides blue. The experimental results indicated that RBITC/Calcein-protocells were hardly internalized by bEnd.3 cells, while the modification of Lf ligand was beneficial to 


\subsection{In Vivo Research}

\subsubsection{Biodistribution} lf-protocells, unmodified protocells and bare MSNs (denoted as Cy5-1f-protocells,

811 Cy5-protocells, Cy5-MSNs, respectively) were qualitatively assessed by

812 luminescence imaging in vivo (Fig. 6A). The results indicated that the injection site 813 (above the right leg of mice) showed strong fluorescence intensity in all experimental 814 groups after injection for $30 \mathrm{~min}$. After $60 \mathrm{~min}$, the fluorescence intensity of 815 1f-protocells group enhanced dramatically in the brain. Interestingly, the brain 816 fluorescence intensity of lf-protocells was further increased, which was much higher 817 than that of bare MSNs and unmodified protocells groups for $120 \mathrm{~min}$. The 818 fluorescence of bare MSNs and unmodified protocells groups concentrated mainly in 819 abdominal cavity, with little or no enrichment in the brain during the whole time of 820 the test.

821 According to the above results, lf-protocells had specific brain-targeting 822 properties in mice, which may be distributed in the brain at a high concentration due 823 to the modification of active targeting Lf ligand. While either bare MSNs or 824 unmodified protocells had no brain targeting. Noteworthily, Lf is a type of ligand with 825 brain-targeting potential, meanwhile, previous studies have demonstrated that the expression of Lf receptor was increased in the brain of PD patients [44]. Hence, the 
high expression of Lf receptor in cerebral microvascular endothelial cells can be utilized to achieve internalization through blood-brain barrier (BBB) by receptor-mediated transport pathway, so as to promote the brain targeting ability of lf-protocells and make them more enriched in the brain of PD patients [45]. Lf-protocells could not only make the brain absorb more levodopa and curcumin, increase the distribution of drugs in the brain compared to free drugs, but also overcome the disadvantage of low bioavailability of curcumin, thus play a better therapeutic role in PD.

3.11.2 L/C-Lf-Pro ameliorated motor function deficits in MPTP-induced PD model mice.

Exposure to MPTP induces a PD-like syndrome such as motor retardation in mice [34]. In the brain, MPTP is converted to $\mathrm{MPP}^{+}$, which is selectively transported into dopaminergic neuron axon terminals, causing oxidative stress, mitochondrial dysfunction, and cell death [46].

The motor function of PD mice was assessed using the open-field test. Our results indicated that motor function was significantly attenuated from day 1 to day 5 in the MPTP-exposed group, compared with the control group $(\mathrm{P}<0.001)$. Neither levodopa nor levodopa/curcumin did affect the improvement of motor function, however, L/C-Pro and L/C-Lf-Pro significantly rescued the reduction of motor function induced by MPTP $(\mathrm{P}<0.05, \mathrm{P}<0.01)$ (Fig. 6B), and the improvement effect of L/C-Lf-Pro were superior to that of L/C-Pro $(\mathrm{P}<0.05)$. 

better therapeutic effect on PD-related motor deficits than other groups. One of the 851 possible reasons is that lf-protocells can package drugs into nanoparticles for 852 elevating the bioavailability of drugs. Especially, lf-protocells have an active brain-targeting distribution, which can deliver more drugs into the brain and exploit the advantages to treat PD. But it is important to note that the behavioral judgement of autonomic activity in mice includes a variety of evaluation systems, one of which is selected in this experiment. At the same time, behavioral evaluation is only a part of PD efficacy at the animal level. Therefore, the PD therapy evaluation of lf-protocells needs to be further completed at the animal level in our study.

Because lf-protocells hold several advantages, including drug loading capacity, stability, and the ease of achieving multi-functionality, next studies will explore further improvement of lf-protocells through the addition of design features such as delivering additional synergistic drug combinations, inclusion of other neuroprotective agents.

Fig. 6 In vivo experiments on biodistribution and improvement of motor function deficits.

(A) In vivo imaging of BALB/c-nu mice after administration of Cy5-MSNs, Cy5-protocells, MPTP-induced PD model mice. Sum of lines crossing in different experimental groups for five days. (mean $\pm \mathrm{SD} ; \mathrm{n}=3$. $\mathrm{ns} \mathrm{P}>0.05, * \mathrm{P}<0.05, * * \mathrm{P}<0.01, * * * \mathrm{P}<0.001$ compared with the 
Cy5-lf protocells; Rot: rotenone; C: curcumin; L: levodopa; Lf-Pro: lf-protocells.

Fig. 7 Schematic illustration of binary-drug loaded lf-protocells for treating Parkinson's

\section{disease.}

(A) Parkinson's disease (PD) is the second most frequent neurodegenerative disease. (B) The pathological hallmark of PD is the characteristic loss of dopaminergic neurons and the appearance of Lewy bodies formed by misfolded and oligomeric $\alpha$-synuclein. Both mitochondrial dysfunction and oxidative stress are key elements in the pathogenesis of PD. (C) The blood-brain barrier (BBB) limits the delivery of the vast majority of drugs passing from the bloodstream into the brain. (D) Drug delivery system named as binary-drug loaded lf-protocells (L/C-Lf-Pro) shows greatly improved BBB transport efficiency of levodopa and curcumin through lactoferrin receptor-mediated endocytosis. (C) While penetrating into the central nervous system, levodopa and curcumin exhibit neuroprotective effects aganist PD with multiple mechanisms, including enhancing the cell viability, decreasing the expression of a-synuclein, ameliorating oxidative stress and mitochondrial dysfunction.

\section{Conclusion}

In summary, this work prepared and characterized successfully organic-inorganic composite nanoparticles (namely lf-protocells) which were formed by coating MSNs with lf-modified lipid bilayer for levodopa/curcumin co-delivery to brain, and evaluated therapeutic effects of the binary-drug loaded lf-protocells on PD both in vitro and in vivo, and explored the possible related mechanism (Fig. 7). As a 
893

894

mitochondrial protective antioxidant, curcumin combined with levodopa was typically effective for PD treatment. It was worth noting that levodopa not only acted as a dopamine precursor, but also showed a certain degree of anti-oxidative activity. It was reported that levodopa oxidation products prevented $\mathrm{H}_{2} \mathrm{O}_{2}$-induced oxidative damage to cellular DNA in cultured tissue cells [47]. Compared to that observed following treatment with free levodopa and curcumin, binary-drug loaded lf-protocells (L/C-Lf-Pro) could increase the distribution and bioavailability of drugs in the brain, thereby exhibit better effects on reducing the level of ROS, increasing the level of SOD and total GSH, ameliorating MMP reduction and decreasing the damage of oxidative stress to PD cells. Furthermore, L/C-Lf-Pro treatment not only reduced $\alpha$-syn accumulation that could cause the formation of Lewy bodies, but also elevated the activity of $\mathrm{TH}$, which is the rate limiting enzyme for the synthesis of dopamine. Presumably, binary-drug loaded lf-protocells (L/C-Lf-Pro) not only converted more levodopa into dopamine to make up for the loss in the brain, but also inhibited MMP reduction, turned off apoptosis signaling pathway, alleviated dopaminergic neuron damage and restored cell survival rate. Loss of dopaminergic neurons leading to striatal dopamine depletion is the core mechanism underlying the cardinal motor features of PD [48]. In vivo, the intraperitoneal injection of L/C-Lf-Pro resulted in improvement of motor function deficits, which was evidently superior to those of any other groups. However, more experiments are required to testify the current point of view. For example, an in vivo study using MPTP-induced animal models is necessary to be further completed. Overall, L/C-Lf-Pro not only replenished the loss of 
dopamine in the brain, but also played the neuroprotective role of antioxidant and repairing injured neurons. The synergy of two different mechanisms can yield a better efficacy for PD, which has been successfully confirmed in vitro and in vivo. As far as we know, it is the first time that the effect of levodopa \& curcumin loaded lf-protocells combination therapy has been applied in PD. The most intriguing thing is the potential application of this approach to other CNS diseases where future lf-protocells could be extended to nanoparticle delivery system for a broad range of drugs, genes or cell-specific targeting [49]. Thus, lf-protocells could offer a basis for future nanocarrier for drug delivery to the CNS.

\section{Declarations}

\section{Ethics approval and consent to participate}

The study was approved by Ethics Committee of Institute of Medicinal Biotechnology, Chinese Academy of Medical Science \& Peking Union Medical College.

\section{Consent for publication}

All authors concur with the submission and publication of this paper.

\section{Competing interests}

The authors declare that they have no competing interests.

\section{Availability of data and materials}

The datasets used and/or analysed during the current study are available from the corresponding author on reasonable request.

\section{Funding}



This work was supported by the CAMS Initiative for Innovative Medicine (Grant No.

938

939

940

941

942

943

944

945

946

947

948

949

950

951

952

953

954

955

956

957

958

959

960 2017-I2M-1-012)

\section{Contributions}

GL and XL led the research. WZ and CL carried out most of experiments and analyzed the data. FY, XN and XM participated in this project. CL and WZ wrote the manuscript, which GL and XL reviewed. All authors read and approved the final manuscript.

\section{Corresponding authors}

Correspondence to GL or XL.

\section{Authors' information}

${ }^{1}$ Wenkai Zhou, Chang Liu contributed equally to this article.

2 Institute of Medicinal Biotechnology, Chinese Academy of Medical Science \& Peking Union Medical College, Beijing, China.

3 Department of Pharmaceutics, School of Pharmaceutical Sciences, Peking University, Beijing, China.

${ }^{4}$ Department of Immunology \& Centre for Immunotherapy, Institute of Basic Medical Sciences, Peking Union Medical College, Chinese Academy of Medical Sciences, Beijing, China.

\section{Reference}

1. Cossu G, Rinaldi R, Colosimo C. The rise and fall of impulse control behavior disorders. Parkinsonism Relat Disord. 2018; 46 Suppl 1S24-S9.

2. Fahn S. The 200-year journey of Parkinson disease: Reflecting on the past and looking towards the future. Parkinsonism Relat Disord. 2018; 46 Suppl 1S1-S5. 
3. Calabrese V, Santoro A, Monti D, Crupi R, Di Paola R, Latteri S, Cuzzocrea S, Zappia M, Giordano J, Calabrese EJ, Franceschi C. Aging and Parkinson's Disease: Inflammaging, neuroinflammation and biological remodeling as key factors in pathogenesis. Free Radic Biol Med. 2018; 11580-91.

4. Dias $\mathrm{V}$, Junn E, Mouradian MM. The role of oxidative stress in Parkinson's disease. Journal of Parkinsons Disease. 2013; 3(4):461-91.

5. Hang L, Thundyil J, Lim KL. Mitochondrial dysfunction and Parkinson disease: a Parkin-AMPK alliance in neuroprotection. Ann N Y Acad Sci. 2015; 135037-47.

6. Mosley RL, Benner EJ, Kadiu I, Thomas M, Boska MD, Hasan K, Laurie C, Gendelman HE. Neuroinflammation, Oxidative Stress and the Pathogenesis of Parkinson's Disease. Clinical Neuroscience Research. 2007; 6(5):261-81.

7. Sharma N, Nehru B. Characterization of the lipopolysaccharide induced model of Parkinson's disease: Role of oxidative stress and neuroinflammation. Neurochemistry International. 2015; 8792-105.

8. Szelechowski M, Bétourné A, Monnet Y, Ferré CA, Thouard A, Foret C, Peyrin JM, Hunot S, Gonzalez-Dunia D. A viral peptide that targets mitochondria protects against neuronal degeneration in models of Parkinson's disease. Nat Commun. 2014; 55181.

9. Wu HM, Li T, Wang ZF, Huang SS, Shao ZQ, Wang K, Zhong HQ, Chen SF, Zhang X, Zhu JH. Mitochondrial DNA variants modulate genetic susceptibility to Parkinson's disease in Han Chinese. Neurobiol Dis. 2018; 11417-23.

10. Franco MR, Viana TF, Biscaia S, Bártolo P. Levodopa Incorporation in Alginate Membranes for Drug Delivery Studies. Advanced Materials Research. 2013; 749423-8.

11. Kestenbaum M, Fahn S. Safety of IPX066, an extended release carbidopa-levodopa formulation, for the treatment of Parkinson's disease. Expert Opin Drug Saf. 2015; 14(5):761-7.

12. Xue Z, Wen Z, Li-da D, Li G, Guan-hua D. Advances in anti-Parkinson's disease drugs and their related pharmacological targets. J Int Pharm Res. 2016; 43(01):87-96.

13. Bollimpelli VS, Kumar P, Kumari S, Kondapi AK. Neuroprotective effect of curcumin-loaded lactoferrin nano particles against rotenone induced neurotoxicity. Neurochem Int. 2016; 9537-45.

14. Harish G, Venkateshappa C, Mythri RB, Dubey SK, Mishra K, Singh N, Vali S, Bharath MM. Bioconjugates of curcumin display improved protection against glutathione depletion mediated oxidative stress in a dopaminergic neuronal cell line: Implications for Parkinson's disease. Bioorg Med Chem. 2010; 18(7):2631-8.

15. Ortiz-Ortiz MA, Morán JM, Ruiz-Mesa LM, Niso-Santano M, Bravo-SanPedro JM, Gómez-Sánchez R, González-Polo RA, Fuentes JM. Curcumin exposure induces expression of the Parkinson's disease-associated leucine-rich repeat kinase 2 (LRRK2) in rat mesencephalic cells. Neurosci Lett. 2010; 468(2):120-4.

16. Jagatha B, Mythri RB, Vali S, Bharath MM. Curcumin treatment alleviates the effects of glutathione depletion in vitro and in vivo: therapeutic implications for Parkinson's disease explained via in silico studies. Free Radic Biol Med. 2008; 44(5):907-17.

17. Kulkarni AP, Kellaway LA, Lahiri DK, Kotwal GJ. Neuroprotection from complement-mediated inflammatory damage. Ann N Y Acad Sci. 2004; 1035147-64.

18. Liu Z, Yu Y, Li X, Ross CA, Smith WW. Curcumin protects against A53T alpha-synuclein-induced toxicity in a PC12 inducible cell model for Parkinsonism. Pharmacol 
1012

1013

1014

1015

1016

1017

1018

1019

1020

1021

1022

1023

1024

1025

1026

1027

1028

1029

1030

1031

1032

1033

1034

1035

1036

1037

1038

1039

1040

1041

1042

1043

1044

1045

1046

1047

1048

Res. 2011; 63(5):439-44.

19. Wang J, Du XX, Jiang $H$, Xie JX. Curcumin attenuates 6-hydroxydopamine-induced cytotoxicity by anti-oxidation and nuclear factor-kappa B modulation in MES23.5 cells. Biochem Pharmacol. 2009; 78(2):178-83.

20. Lee WH, Loo CY, Bebawy M, Luk F, Mason RS, Rohanizadeh R. Curcumin and its derivatives: their application in neuropharmacology and neuroscience in the 21st century. Curr Neuropharmacol. 2013; 11(4):338-78.

21. Monroy A, Lithgow GJ, Alavez S. Curcumin and neurodegenerative diseases. Biofactors. 2013; 39(1):122-32.

22. Butler KS, Durfee PN, Theron C, Ashley CE, Carnes EC, Brinker CJ. Protocells: Modular Mesoporous Silica Nanoparticle-Supported Lipid Bilayers for Drug Delivery. Small. 2016; 12(16):2173-85.

23. Liu J, Stace-Naughton A, Jiang $X$, Brinker CJ. Porous nanoparticle supported lipid bilayers (protocells) as delivery vehicles. J Am Chem Soc. 2009; 131(4):1354-5.

24. Ashley CE, Carnes EC, Phillips GK, Padilla D, Durfee PN, Brown PA, Hanna TN, Liu J, Phillips B, Carter $\mathrm{MB}$, et al. The targeted delivery of multicomponent cargos to cancer cells by nanoporous particle-supported lipid bilayers. Nat Mater. 2011; 10(5):389-97.

25. Chen F, Hong H, Zhang Y, Valdovinos HF, Shi S, Kwon GS, Theuer CP, Barnhart TE, Cai W. In vivo tumor targeting and image-guided drug delivery with antibody-conjugated, radiolabeled mesoporous silica nanoparticles. ACS Nano. 2013; 7(10):9027-39.

26. Mekaru H, Lu J, Tamanoi F. Development of mesoporous silica-based nanoparticles with controlled release capability for cancer therapy. Adv Drug Deliv Rev. 2015; 9540-9.

27. Meng $H$, Mai WX, Zhang $H$, Xue $M$, Xia T, Lin S, Wang X, Zhao $Y$, Ji Z, Zink Jl, Nel AE. Codelivery of an optimal drug/siRNA combination using mesoporous silica nanoparticles to overcome drug resistance in breast cancer in vitro and in vivo. ACS Nano. 2013; 7(2):994-1005.

28. Ye $Y$, Sun $Y$, Zhao $H$, Lan M, Gao F, Song $C$, Lou $K, L i ~ H$, Wang W. A novel lactoferrin-modified beta-cyclodextrin nanocarrier for brain-targeting drug delivery. Int J Pharm. 2013; 458(1):110-7.

29. C B. Physiology: Double function at the blood-brain barrier. Nature. 2014; 509(7501):432-3.

30. BA F, N N, P D, G S, A M-P, A P, B L, N K, JJ H, Y A. Expression of lactoferrin receptors is increased in the mesencephalon of patients with Parkinson disease. Proceedings of the National Academy of Sciences of the United States of America. 1995; 92(21):9603-7.

31. T K, I T, T Y, DG W, PL M. Lactotransferrin immunocytochemistry in Alzheimer and normal human brain. The American journal of pathology. 1993; 142(5):1574-85.

32. Zhai H, Wang $Y$, Wang M, Liu S, Yu F, Gao C, Li G, Wu Q. Construction of a Glutathione-Responsive and Silica-Based Nanocomposite for Controlled Release of Chelator Dimercaptosuccinic Acid. Int J Mol Sci. 2018; 19(12):E3790.

33. Meng H, Wang M, Liu H, Liu X, Situ A, Wu B, Ji Z, Chang CH, Nel AE. Use of a lipid-coated mesoporous silica nanoparticle platform for synergistic gemcitabine and paclitaxel delivery to human pancreatic cancer in mice. ACS Nano. 2015; 9(4):3540-57.

34. Li Y, Perry T, Kindy MS, Harvey BK, Tweedie D, Holloway HW, Powers K, Shen H, Egan JM, Sambamurti K, et al. GLP-1 receptor stimulation preserves primary cortical and dopaminergic neurons in cellular and rodent models of stroke and Parkinsonism. Proc Natl Acad Sci USA. 2009; 106(4):1285-90. 
35. Kim MH, Na HK, Kim YK, Ryoo SR, Cho HS, Lee KE, Jeon H, Ryoo R, Min DH. Facile synthesis of monodispersed mesoporous silica nanoparticles with ultralarge pores and their application in gene delivery. ACS Nano. 2011; 5(5):3568-76.

36. Roggers RA, Joglekar M, Valenstein JS, Trewyn BG. Mimicking red blood cell lipid membrane to enhance the hemocompatibility of large-pore mesoporous silica. ACS Appl Mater Interfaces. 2014; 6(3):1675-81.

37. Kundu P, Das M, Tripathy K, Sahoo SK. Delivery of Dual Drug Loaded Lipid Based Nanoparticles across the Blood-Brain Barrier Impart Enhanced Neuroprotection in a Rotenone Induced Mouse Model of Parkinson's Disease. ACS Chem Neurosci. 2016; 7(12):1658-70.

38. Chen $\mathrm{W}, \mathrm{Su} \mathrm{H}, \mathrm{Xu} \mathrm{Y}$, Jin $\mathrm{C}$. In vitro gastrointestinal digestion promotes the protective effect of blackberry extract against acrylamide-induced oxidative stress. Sci Rep. 2017; 740514.

39. Perricone C, De Carolis C, Perricone R. Glutathione: a key player in autoimmunity. Autoimmun Rev. 2009; 8(8):697-701.

40. Martinou J-C, Youle Richard J. Mitochondria in Apoptosis: Bcl-2 Family Members and Mitochondrial Dynamics. Developmental Cell. 2011; 21(1):92-101.

41. Circu ML, Aw TY. Reactive oxygen species, cellular redox systems, and apoptosis. Free Radical Biology and Medicine. 2010; 48(6):749-62.

42. Bock FJ, Tait SWG. Mitochondria as multifaceted regulators of cell death. Nature reviews Molecular cell biology. 2020; 21(2):85-100.

43. Skommer J, Brittain T, Raychaudhuri S. Bcl-2 inhibits apoptosis by increasing the time-to-death and intrinsic cell-to-cell variations in the mitochondrial pathway of cell death Apoptosis. 2010; 15(10):1223-33.

44. Huang RQ, Ke WL, Qu YH, Zhu JH, Pei YY, Jiang C. Characterization of lactoferrin receptor in brain endothelial capillary cells and mouse brain. J Biomed Sci. 2007; 14(1):121-8.

45. Fang F, Zou D, Wang W, Yin Y, Yin T, Hao S, Wang B, Wang G, Wang Y. Non-invasive approaches for drug delivery to the brain based on the receptor mediated transport. Mater Sci Eng C Mater Biol Appl. 2017; 761316-27.

46. Javitch JA, D'Amato RJ, Strittmatter SM, Snyder SH. Parkinsonism-inducing neurotoxin, $\mathrm{N}$-methyl-4-phenyl-1,2,3,6 -tetrahydropyridine: uptake of the metabolite $\mathrm{N}$-methyl-4-phenylpyridine by dopamine neurons explains selective toxicity. Proc Natl Acad Sci USA. 1985; 82(7):2173-7.

47. Shi Y-I, Benzie IFF, Buswell JA. L-DOPA oxidation products prevent $\mathrm{H} 2 \mathrm{O} 2$-induced oxidative damage to cellular DNA. Life sciences. 2002; 71(26):3047-57.

48. Poewe W, Seppi K, Tanner CM, Halliday GM, Brundin P, Volkmann J, Schrag AE, Lang AE. Parkinson disease. Nat Rev Dis Primers. 2017; 317013.

49. Dengler EC, Liu J, Kerwin A, Torres S, Olcott CM, Bowman BN, Armijo L, Gentry K, Wilkerson J, Wallace J, et al. Mesoporous silica-supported lipid bilayers (protocells) for DNA cargo delivery 
A
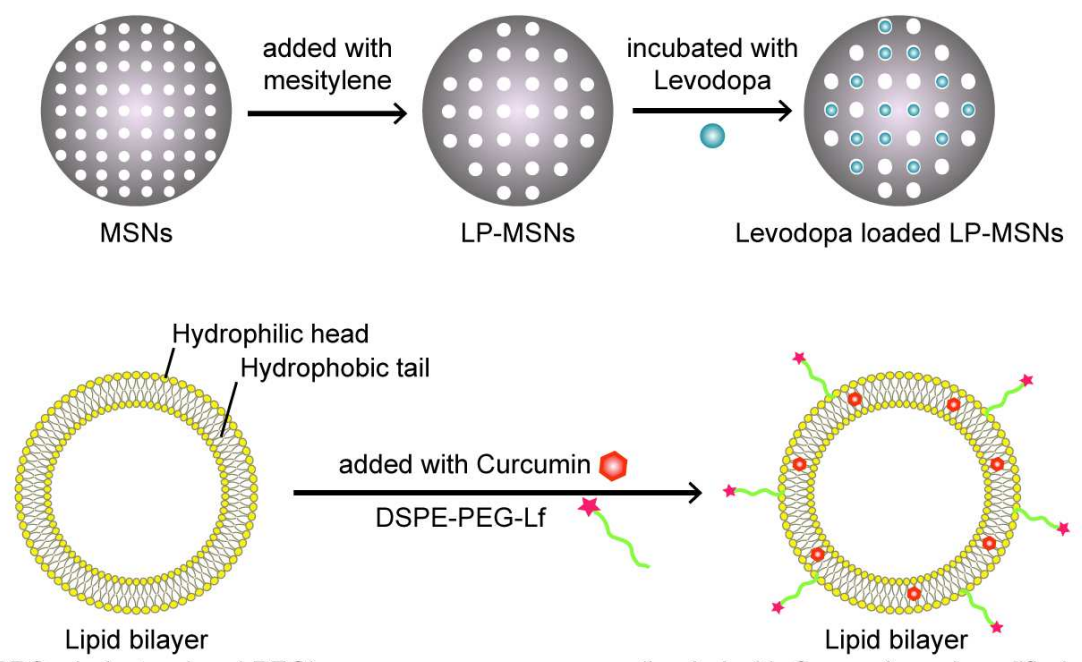

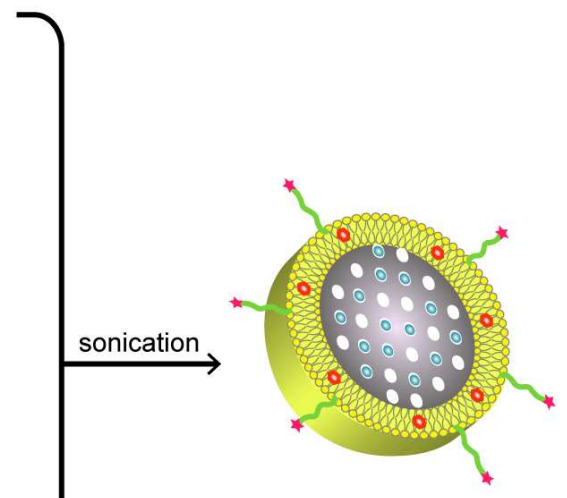

Lf-Protocells (loaded with Levodopa/Curcumin and modified with Lf)

(DPPC, cholesterol and PEG)

(loaded with Curcumin and modified with Lf)

B

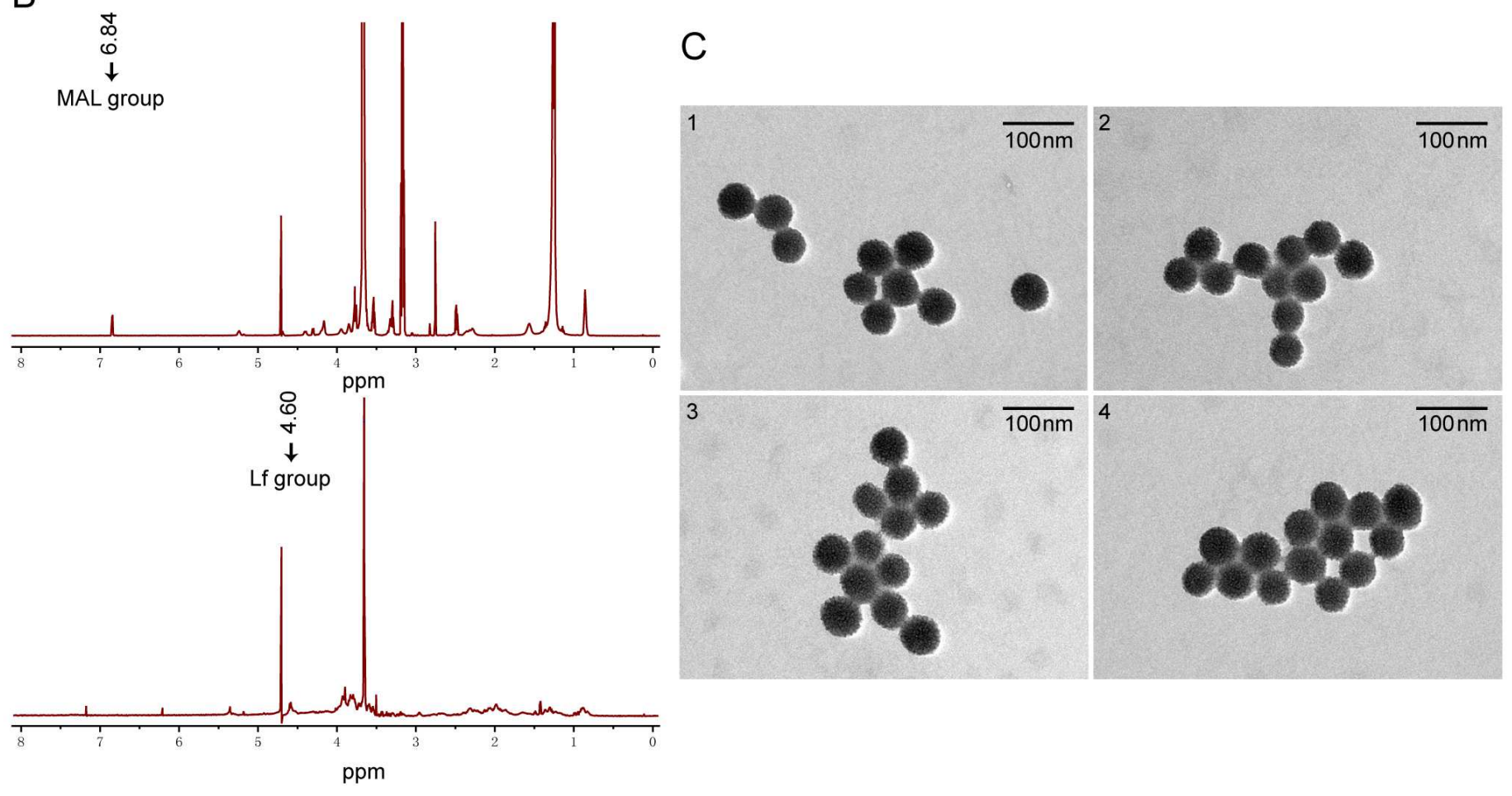

Figure 1

Preparation and characterization of If-protocells. 

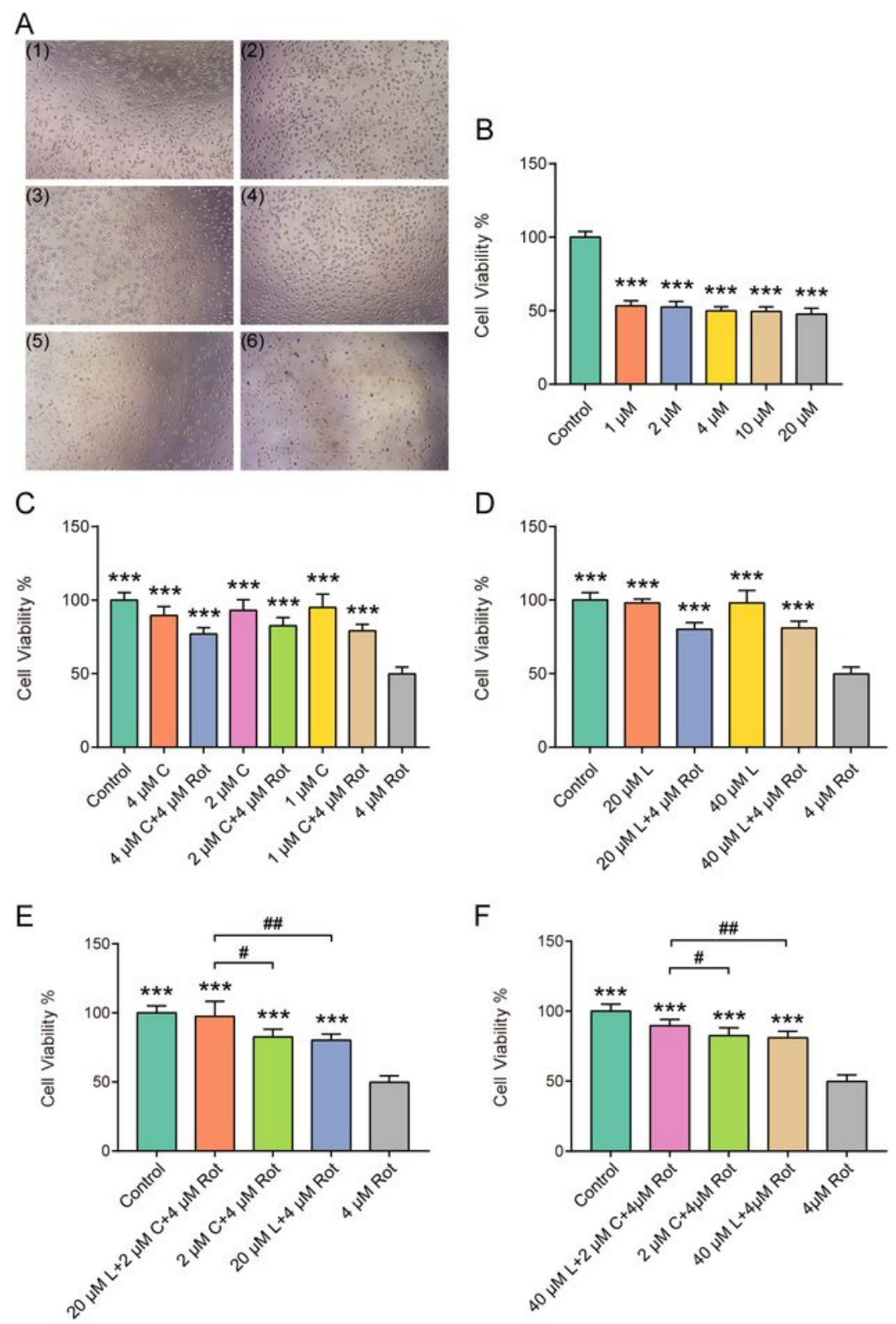

Figure 2

Effects of levodopa and curcumin on Rot-induced cytotoxicity. 

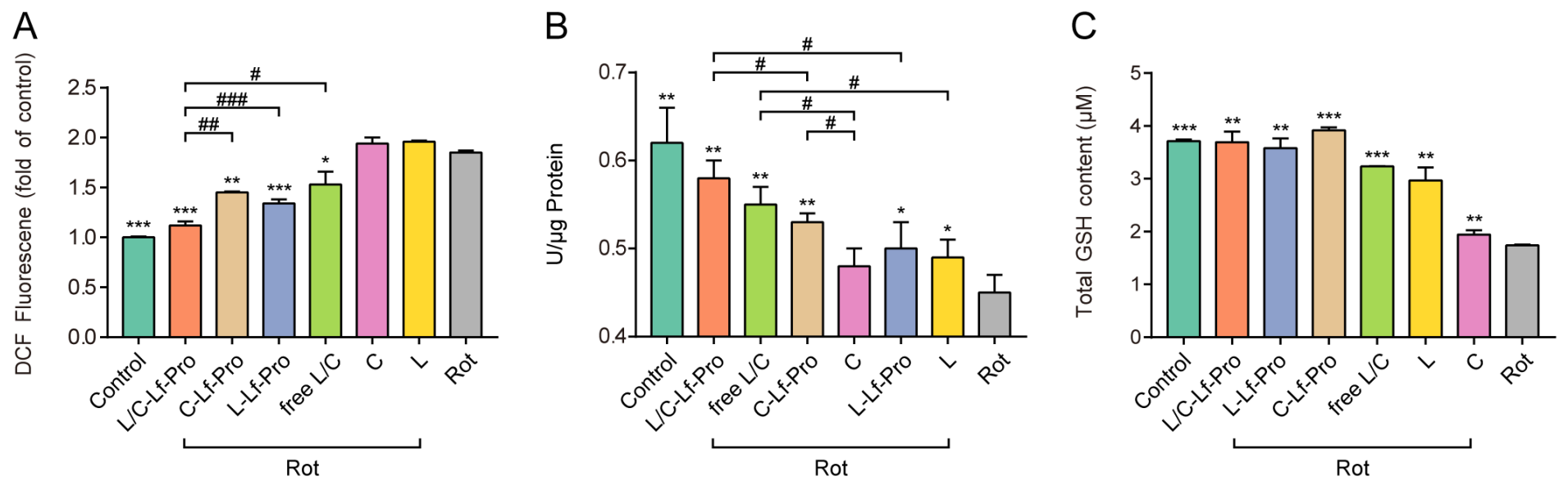

\section{Figure 3}

Lf-protocells treatment ameliorated oxidative stress in SH-SY5Y cells induced by Rot.

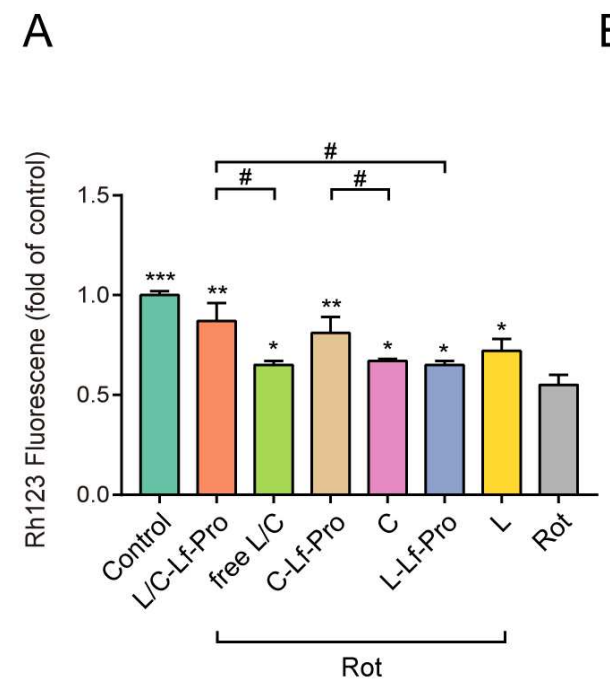

$\mathrm{B}$
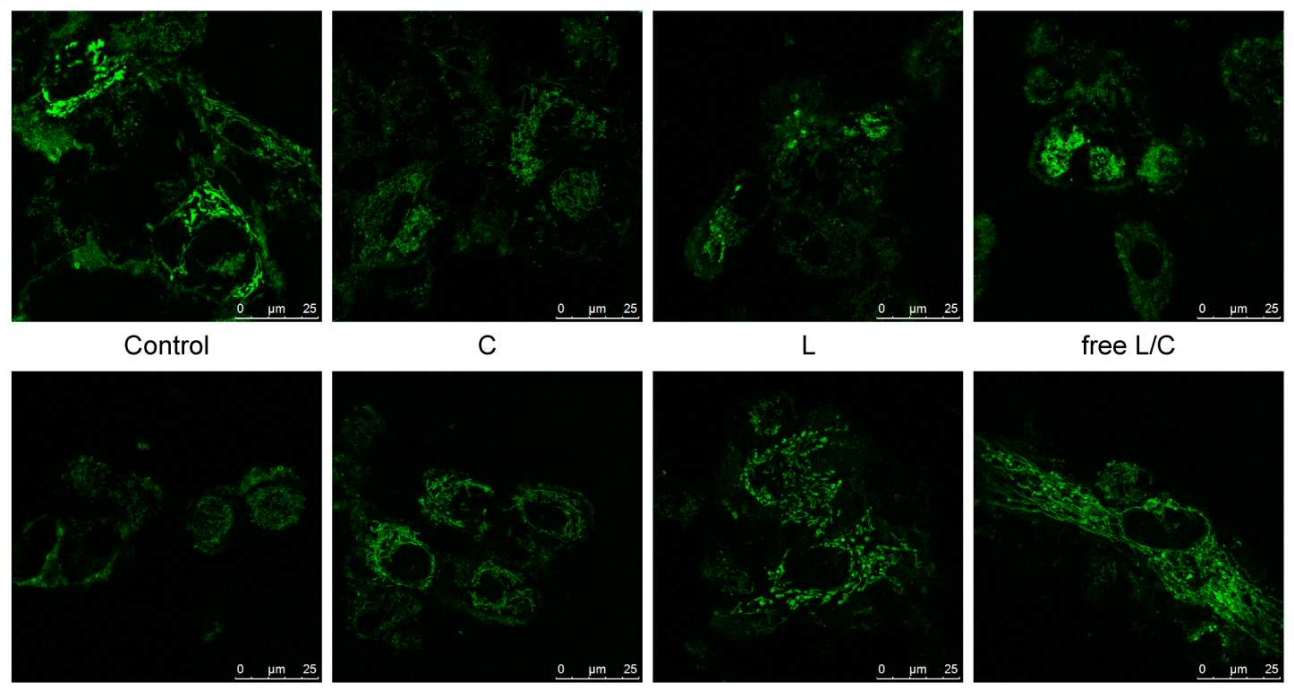

free $L / C$

Rot

C-Lf-Pro

L-Lf-Pro

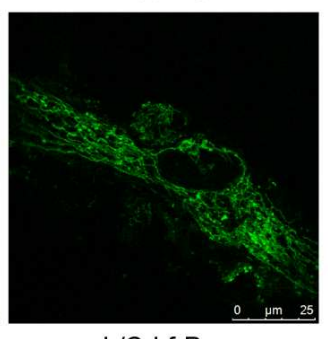

L/C-Lf-Pro

Figure 4

Lf-protocells suppressed Rot-induced mitochondrial dysfunction in SH-SY5Y cells. 

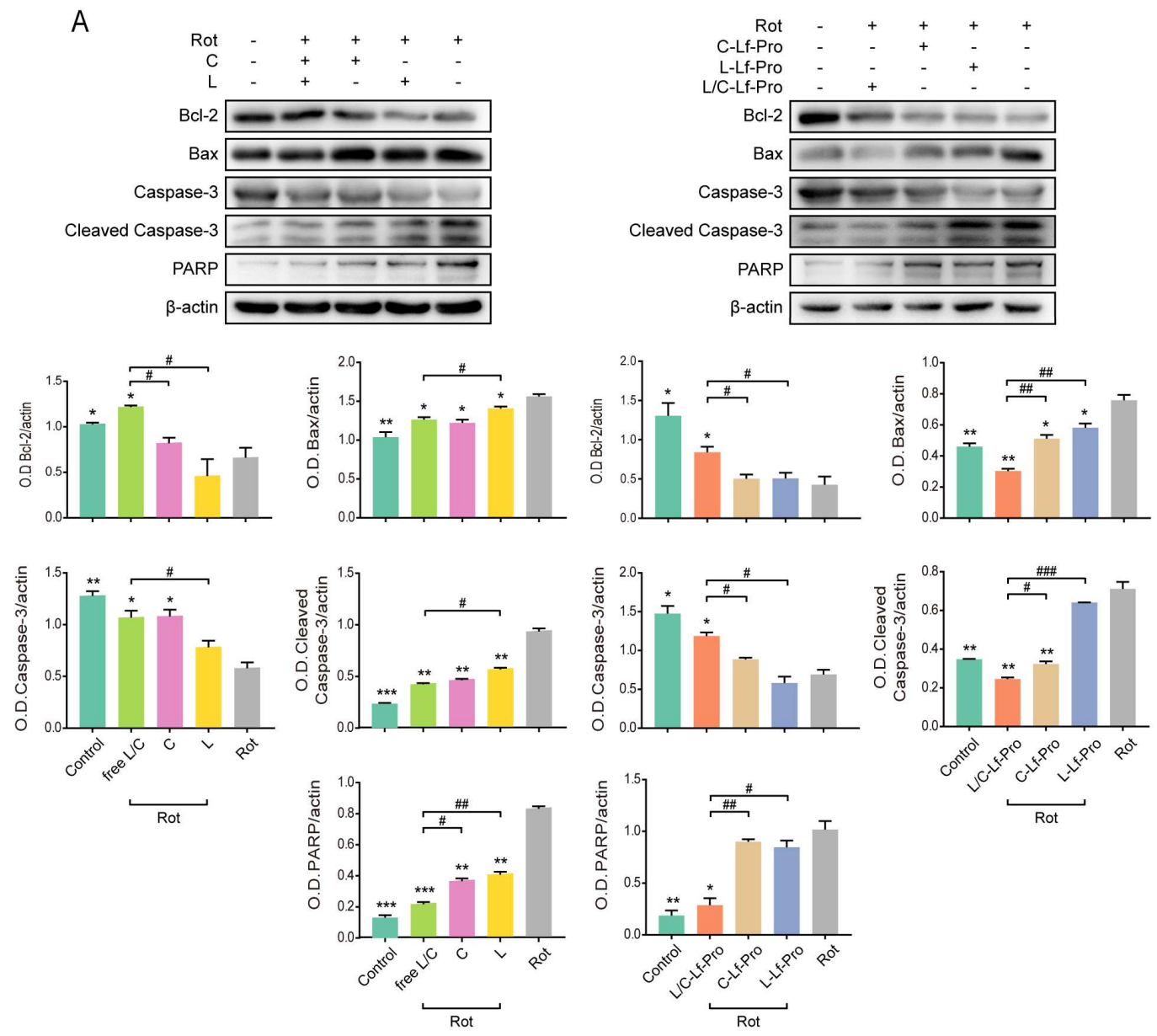

B
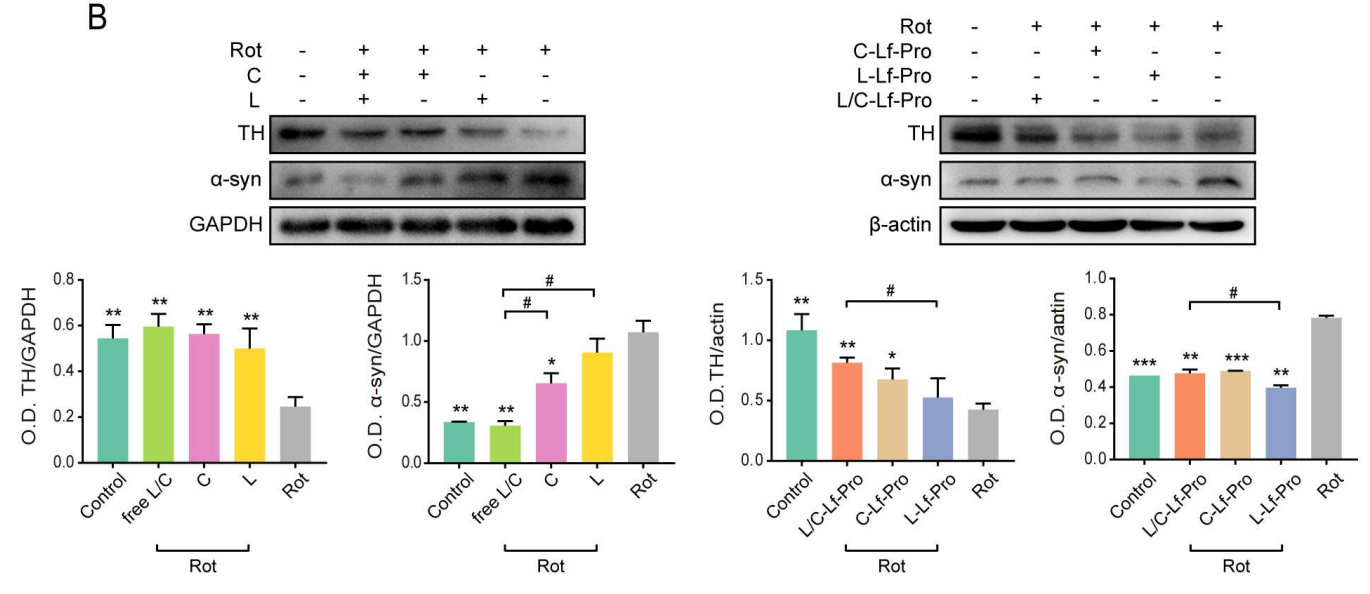

\section{Figure 5}

The effects of levodopa/curcumin and L/C-Lf-Pro on rotenone-induced variation in expression of apoptosis related proteins and PD related proteins through western blot analysis. 


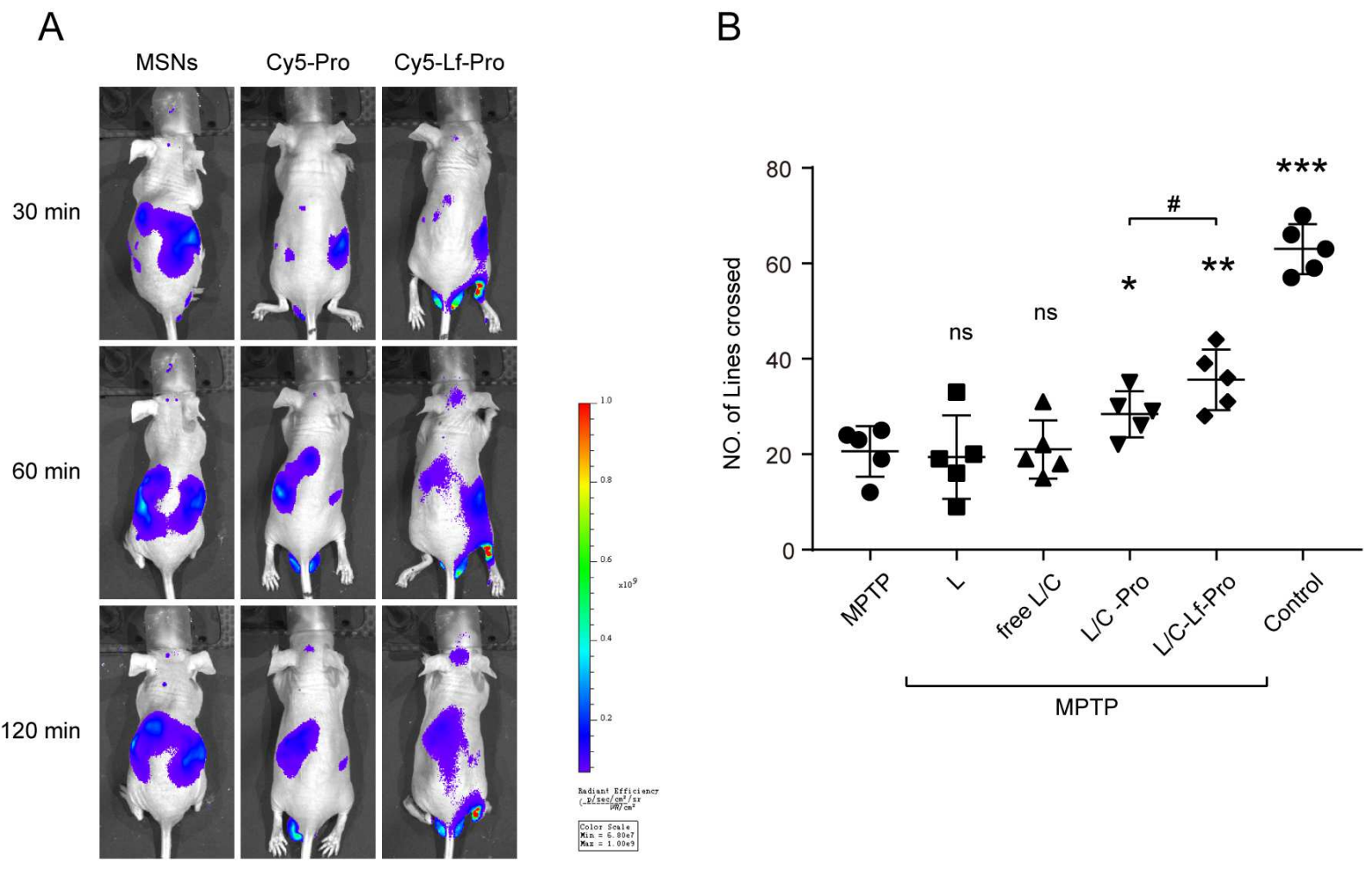

Figure 6

In vivo experiments on biodistribution and improvement of motor function deficits. 


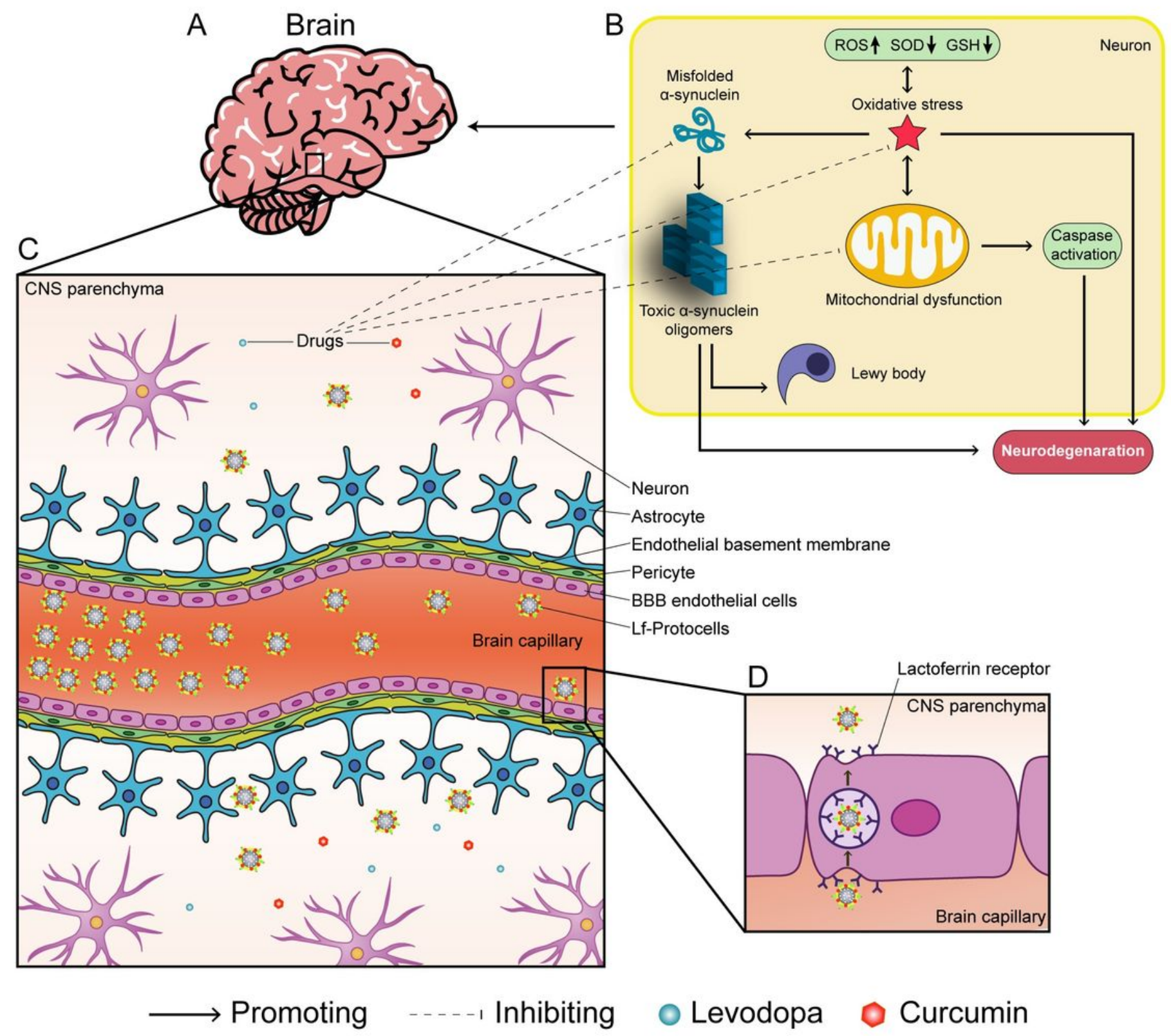

Figure 7

Schematic illustration of binary-drug loaded If-protocells for treating Parkinson's disease.

\section{Supplementary Files}

This is a list of supplementary files associated with this preprint. Click to download.

- supplement.docx 